A. Morimoto

Nagoya Math. J.

Vol. 38 (1970), 153-179

\title{
PROLONGATIONS OF G-STRUGTURES TO TANGENT BUNDLES OF HIGHER ORDER
}

\author{
AKIHIKO MORIMOTO
}

\section{To Professor Katuzi Ono on the occasion of his 60th birthday}

\section{$\S$ Introduction and Notations.}

In the previous paper [4] we have studied the prolongations of $G$ structures to tangent bundles. The purpose of the present paper is to generalize the previous prolongations and to look at them from a wide view as a special case by considering the tangent bundles of higher order. In fact, in some places, the arguments and calculations in [4] are more or less simplified. Since the usual tangent bundle $T(M)$ of a manifold $M$ considers only the first derivatives or first contact elements of $M$, the previous paper contains, in most parts, only the calculation of derivatives of first order.

Now, since the tangent bundle $\stackrel{r}{T} M$ to a manifold $M$ of order $r$ concerns with the derivatives of higher order (up to order $r$ ), the situations should be much complicated. Nevertheless, the (covariant) functor $\stackrel{r}{T}: M \rightarrow$ $\stackrel{r}{T} M$ from the category of differentiable manifolds and differentiable maps to the same category, fortunately, has many properties similar to the functor $T: M \rightarrow T M$. For instance, (i) $\stackrel{r}{T} G$ is a Lie group if $G$ is a Lie group, (ii) $\stackrel{r}{T} R^{n}$ has a natural vector space structure and (iii) $\stackrel{r}{T} G L(n)$ can be considered as a Lie subgroup of $G L(n(r+1))$. Therefore, we can follow the procedure in [4] by replacing the functor $T$ with the functor $\stackrel{r}{T}$.

We mention here that Yano and Ishihara [7] study the prolongations of tensor fields to the tangent bundles of order 2 from the viewpoint of tensor analysis.

In $\$ 1$, we explain the notion of tangent bundles $\stackrel{r}{T} M$ of order $r$ to a manifold $M$, tangent bundles of order 1 coinciding with the usual tangent bundle.

Received January 20, 1969 
In $\S 2,3$, we consider the tangent bundles to a Lie group of order $r$ and prove that if a Lie group $G$ operates on a manifold $M$ effectively then the Lie group $\stackrel{r}{T} G$ operates canonically on $\stackrel{r}{T} M$ also effectively.

In $\S 4,5$, we consider the vector space $\stackrel{r}{T} R^{n}$ and prove that $\stackrel{r}{T} G L(n)$ operates on $\stackrel{r}{T} R^{n}$ as a linear transformation group.

In $\S 6$, we consider the tangent bundle of higher order to (principal) fibre bundles.

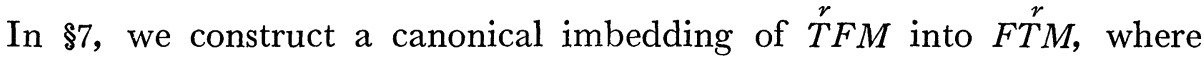
$F M$ denotes the frame bundle of $M$. Using the results in $\$ 6,7$ we can define in $\S 8$ the prolongation $P^{(r)}$ of order $r$ of a $G$-structure $P$ to the tangent bundle $\stackrel{r}{T} M$ for any $r$.

In $\S 9$, we prove that a diffeomorphism $\Phi: M \rightarrow M^{\prime}$ is an isomorphism of $G$-structures $P$ with $P^{\prime}$ if and only if $\stackrel{r}{T} \Phi$ is an isomorphism of $P^{(r)}$ with $P^{(r)}$.

In $\$ 10$, we prove that a $G$-structure $P$ is integrable if and only if the prolongation $P^{(r)}$ is integrable.

In $\$ 11$, we consider some classical $G$-structures and prove, among others, that if a manifold $M$ has an (resp. an integrable) almost complex sturcture, symplectic structure, pseudo-Riemannian structure or a (completely integrable) differential system, then $\stackrel{r}{T} M$ has canonically the same kind of structures. Moreover, if $M$ has an almost contact structure, then $\stackrel{r}{T} M$ has a canonical almost complex structure for $r$ odd and has an almost contact structure for $r$ even.

In this paper, all manifolds and mappings (functions) are assumed to be differentiable of class $C^{\infty}$, unless otherwise stated. If $\varphi: M \rightarrow N$ is a map of a set $M$ into a set $N$ and if $A$ is a subset of $M$, we often denote by $\varphi$ itself the restriction $\varphi \mid A$ of $\varphi$ to $A$, if there is no confusion. If $\varphi_{i}$ : $M_{i} \rightarrow N_{i}$ is a map for $i=1,2$, then the map $\varphi_{1} \times \varphi_{2}: M_{1} \times M_{2} \rightarrow N_{1} \times N_{2}$ is defined by $\left(\varphi_{1} \times \varphi_{2}\right)\left(x_{1}, x_{1}\right)=\left(\varphi_{1}\left(x_{1}\right), \varphi_{2}\left(x_{2}\right)\right)$ for $x_{i} \in M_{i}, i=1,2$. If $M_{1}=M_{2}=M$, the map $\left(\varphi_{1}, \varphi_{2}\right): M \rightarrow N_{1} \times N_{2}$ is defined by $\left(\varphi_{1}, \varphi_{2}\right)(x)=\left(\varphi_{1}(x), \varphi_{2}(x)\right)$ for $x \in M$.

In the following; $R^{n}$ denotes always the $n$-dimensional real number space. The group of all linear automorphisms of $R^{n}$ will be denoted by $G L(n, R)$ or simply by $G L(n)$. If $a_{j}^{i} \in R$ for $i, j=1,2, \cdots, n$, we denote by $\left(a_{j}^{i}\right)$ the matrix of degree $n$ whose $(i, j)$-entry is $a_{j}^{i}$. 


\section{\$1. Tangent bundles of order $r$.}

Let $\mathfrak{F}$ be the set of all real valued differentiable functions defined on some neighborhood of $R$ containing zero. Take two functions $f$ and $g$ in $\mathfrak{F}$. For a positive integer $r$ we say $f$ is $r$-equivalent to $g$ iff $d^{\nu} f / d t^{\nu}=$ $d^{\nu} g / d t^{\nu}$ at $t=0$ for $\nu=0,1, \cdots, r$, and we will denote it by $f_{r} g$. The relation $\widetilde{r}$ is clearly an equivalence relation in $\mathfrak{乛}$. Let $M$ be an $n$-dimensional manifold, and let $C^{\infty}(M)$ be the ring of all differentiable functions defined on $M$. We denote by $\widetilde{S}(M)$ (resp. $S(M)$ ) the set of all maps $\bar{\varphi}$ of some open interval $(-\varepsilon, \varepsilon)$ (resp. $R$ ) into $M, \infty \geqq \varepsilon>0$ depending on $\varphi$. Let $\varphi$ and $\psi$ be two maps of $\widetilde{S}(M)$. We say that $\varphi$ is $r$-equivalent to $\psi$ iff $f \circ \varphi \widetilde{r} f \circ \psi$ for every $f \in C^{\infty}(M)$ and denote it by $\varphi \underset{r}{\sim} \psi$. The relation $\widetilde{r}$ is also an equivalence relation in $\widetilde{S}(M)$. For $\varphi \in \widetilde{S}(M)$ we denote by $[\varphi]_{r}$ the equivalence class in $\widetilde{S}(M)$ containing $\varphi$.

Definition 1. 1. We call $[\varphi]_{r}$ the $r$-tangent to $M$ at $p \in M$ (or $r$-jet) defined by $\varphi$ iff $\varphi(0)=p$.

For any $r$-tangent $[\varphi]_{r}$ to $M$ there exists $\varphi^{\prime} \in S(M)$ such that $\left[\varphi^{\prime}\right]_{r}=[\varphi]_{r}$ by virtue of the following

Lemma 1. 2. Let $\varphi \in \widetilde{S}(M)$. Then there exist some $\varepsilon_{1}>0$ and $\varphi^{\prime} \in S(M)$ such that $\varphi$ is defined on $\left(-\varepsilon_{1}, \varepsilon_{1}\right)$ and $\varphi(t)=\varphi^{\prime}(t)$ for $|t|<\varepsilon_{1}$.

Proof. Since $\varphi \in \widetilde{S}(M)$, there is some $\varepsilon>0$ such that $\varphi$ is defined on $(-\varepsilon, \varepsilon)$. We can find a function $g \in C^{\infty}(R)$ such that $g(t)=t$ for $|t| \leq \varepsilon / 2$ and $g(t)=0$ for $|t| \geqq 2 \varepsilon / 3$ and that $|g(t)| \leq 2 \varepsilon / 3$ for all $t \in R$. Put $\varepsilon_{1}=\varepsilon / 2$ and $\varphi^{\prime}=\varphi \circ g$. It is now clear that $\varphi^{\prime}$ and $\varepsilon_{1}$ satisfy the required conditions.

Q.E.D.

DeEfintion 1. 3. Let $\stackrel{r}{T}(M)$ (or $\stackrel{r}{T} M$ ) be the set of all $r$-tangents to $M$, and for $p \in M$ let $\stackrel{r}{T}_{p}(M)$ be the set of all $r$-tangents to $M$ at $p$. We define $\stackrel{r}{\pi}: \stackrel{r}{T}(M) \rightarrow M$ by ${ }^{r}\left([\varphi]_{r}\right)=\varphi(0)$ for $[\varphi]_{r} \in \stackrel{r}{T}(M)$.

The notion of 1-tangents to $M$ at $p$ coincides with the notion of usual tangent vectors to $M$ at $p$. In order to define the manifold structure in $\stackrel{r}{T} M$ we shall prove the following

Lemma 1. 4. Let $\left\{x_{1}, x_{2}, \cdots, x_{n}\right\}$ be a local coordinate system on some neighborhood $U$ of $p \in M$. Take two elements $\varphi$ and $\psi$ in $S(M)$ such that 
$\varphi(0)=\psi(0)=p$. Then $\varphi \underset{r}{\sim} \psi$ if and only if $x_{i} \circ \varphi \widetilde{r}_{\boldsymbol{r}} \circ \psi$ for $i=1,2, \cdots, n$.

Proof. Suppose $\varphi \underset{r}{\sim} \psi$. There exist a neighborhood $V$ of $p$ contained in $U$ and a function $f_{i} \in C^{\infty}(M)(i=1,2, \cdots, n)$ such that $f_{i}\left|V=x_{i}\right| V$. Since $f_{i} \circ \varphi \underset{r}{\sim} f_{i} \circ \psi$ and since $x_{i} \circ \varphi(t)=f_{i} \circ \varphi(t), \quad x_{i} \circ \psi(t)=f_{i} \circ \psi(t)$ for $|t|<\varepsilon$ with some $\varepsilon>0$, we have $x_{i} \mid \varphi \underset{r}{\sim} x_{i} \circ \psi$ for $i=1,2, \cdots, n$.

Conversely, suppose $x_{i} \circ \varphi \widetilde{r}_{i} \circ \psi$ for $i=1,2, \cdots, n$. Take $f \in C^{\infty}(M)$. We have to prove $f \circ \varphi \sim f \circ \psi$, i.e. $d^{\nu}(f \circ \varphi) / d t^{\nu}=d^{\nu}(f \circ \Psi) / d t^{\nu}$ at $t=0$ for $\nu=0,1,2, \cdots, r$. This holds for $\nu=0$, since $\varphi(0)=\psi(0)$. Define $\Psi: U \rightarrow R^{n}$ by $\Psi(q)=\left(x_{1}(q), x_{2}(q), \cdots, x_{n}(q)\right)$ for $q \in U$. Then the function $F=f \circ \Psi^{-1}$ is an element of $C^{\infty}(\Psi(U))$ and we have $f(q)=F\left(x_{1}(q), \cdots, x_{n}(q)\right)$ for $q \in U$. Since $f(\varphi(t))=F\left(x_{1}(\varphi(t)), \cdots, x_{n}(\dot{\varphi}(t))\right.$, we have the following

$$
\frac{d(f \circ \varphi)}{d t}=\sum_{i=1}^{n}\left[\frac{\partial F}{\partial x_{i}}\right]_{x=\Psi \varphi(t))} \cdot \frac{d\left(x_{i} \circ \varphi\right)}{d t},
$$

and hence we get

$$
\left[\frac{d(f \circ \varphi)}{d t}\right]_{t=0}=\sum_{i=1}^{n}\left[\frac{\partial F}{\partial x_{i}}\right]_{x=\Psi(p)} \cdot\left[\frac{d\left(x_{i} \circ \varphi\right)}{d t}\right]_{t=0} \cdot
$$

Similarly, we have

$$
\left[\frac{d(f \circ \psi)}{d t}\right]_{t=0}=\sum_{i=1}^{n}\left[\frac{\partial F}{\partial x_{i}}\right]_{x=\Psi(p)} \cdot\left[\frac{d\left(x_{i} \circ \psi\right)}{d t}\right]_{t=0} \cdot
$$

Hence we obtain $[d(f \circ \varphi) / d t]_{0}=[d(f \circ \psi) / d t]_{0}$. Differentiate (1. 1) and evaluate at $t=0$, then we get $\left[d^{2}(f \circ \varphi) / d t^{2}\right]_{0}=\left[d^{2}(f \circ \psi) / d t^{2}\right]_{0}$ and so on. Thus we see $f \circ \varphi \underset{r}{\sim} f \circ \psi$.

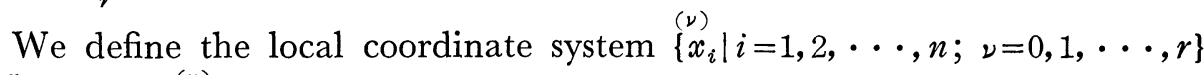
on $\stackrel{r}{(\pi)}^{-1}(U)$ by ${ }^{(\nu)} x_{i}\left([\varphi]_{r}\right)=(1 / \nu !)\left[d^{\nu}\left(x_{i}(\varphi(t))\right) / d t^{\nu}\right]_{t=0}$ for $[\varphi]_{r} \in(\pi)^{-1}(U)$.

It is straightforward to see that $\stackrel{r}{T(M)}$ has a differentiable manifold structure by these coordinate systems and to see that $\stackrel{r}{\pi}$ is a differentiable surjective map of maximal rank. It is also clear that $T_{p}^{r}(M)$ is diffeomorphic to $R^{r n}$ for any $p \in M$.

Definition 1. 5. The manifold $T M$ with the projection $\pi$ is called the tangent bundle to $M$ of order $r$. If $U$ is an open subset of $M$, then $(\pi)^{-1}(U)$ is an open submanifold of $\stackrel{r}{T}(M)$ which can be identified with $\stackrel{r}{T}(U)$. 
However, it must be noticed that $\stackrel{r}{T}(M)(M, \stackrel{r}{\pi})$ is not a vector bundle over $M$.

We define $\pi_{s}^{r}: \stackrel{r}{T}(M) \rightarrow \stackrel{s}{T}(M)$ for $r>s$ by $\pi_{s}^{r}\left([\varphi]_{r}\right)=[\varphi]_{s}$ for $\varphi \in S(M)$.

On the other hand, $M$ can be imbedded in $\stackrel{r}{T(M)}$ by $x \rightarrow\left[r_{x}\right]_{r}$ for $x \in M$, where $\gamma_{x} \in S(M)$ is defined by $\gamma_{x}(t)=x$ for $t \in R$.

Let $N$ be another manifold of dimension $m$. For any map $\Phi: M \rightarrow N$, we define the induced map $\stackrel{r}{T} \Phi: \stackrel{r}{T} M \rightarrow \stackrel{r}{T} N$ by $(\stackrel{r}{T} \Phi)\left([\varphi]_{r}\right)=[\Phi \circ \varphi]_{r}$ for $\varphi \in S(M)$. It is easy to see that $\stackrel{r}{T \Phi}$ is well-defined and that $\stackrel{r}{T \Phi}$ is a differentiable map of $\stackrel{r}{T} M$ into $\stackrel{r}{T} N$. We shall call $\stackrel{r}{T} \Phi$ the tangent to $\Phi$ of order $r$ (or simply $r$-tangent to $\Phi$ ).

Let $\pi_{1}$ (resp. $\left.\pi_{2}\right)$ be the projection of $M \times N$ onto $M$ (resp. $\left.N\right)$. We can readily see that $\stackrel{r}{T}(M \times N)$ can be identified with $\stackrel{r}{T} M \times \stackrel{r}{T} N$ by $[\varphi]_{r} \rightarrow$ $\left(\left[\pi_{1} \circ \varphi\right]_{r},\left[\pi_{2} \circ \varphi\right]_{r}\right)$ for $\varphi \in S(M \times N)$.

We can prove the following Propositions 1.6 and 1. 7 whose proof will be straightforward.

Proposition 1. 6. Let $M_{0}, M_{1}, M_{2}, M_{3}$ be manifolds. and let $\Phi: M_{0} \rightarrow M_{1}$, $\Phi_{1}: M_{1} \rightarrow M_{2}, \Phi^{\prime}: M_{0} \rightarrow M_{2}$ and $\Psi: M_{2} \rightarrow M_{3}$ be maps. Then, we have the following equalities:

(i) $\quad \stackrel{r}{T}\left(\Phi_{1} \circ \Phi\right)=\left(\stackrel{r}{T} \Phi_{1}\right) \circ(\stackrel{r}{T} \Phi)$,

(ii) $\quad \stackrel{r}{T}\left(\Phi, \Phi^{\prime}\right)=\left(\stackrel{r}{T} \Phi, \stackrel{r}{T} \Phi^{\prime}\right)$,

(iii) $\quad \stackrel{r}{T}(\Phi \times \Psi)=\stackrel{r}{T} \Phi \times \stackrel{r}{T} \Psi$,

(iv) $\quad \stackrel{r}{T}\left(1_{M}\right)=1_{T_{M}}$,

where $1_{M}$ stands for the identity map of $M$.

Proposition 1. 7. Let $\pi_{1}\left(\right.$ resp. $\left.\pi_{2}\right)$ be the projection of $M_{1} \times M_{2}$ onto $M_{1}$ (resp. $M_{2}$ ), and let $\tilde{\pi}_{1}$ (resp. $\tilde{\pi}_{2}$ ) be the projection of $\stackrel{r}{T} M_{1} \times \stackrel{r}{T} M_{2}$ onto $\stackrel{r}{T} M_{1}$ (resp. $\left.\stackrel{r}{T} M_{2}\right)$. Then, we have $\stackrel{r}{T}_{\pi_{i}}=\tilde{\pi}_{i}$ for $i=1,2$.

Proposition 1. 8. Let $M, N$ be manifolds and let $\Phi$ be a map of $M$ into $N$ of maximal rank. Then, $\stackrel{r}{T} \Phi$ is a map of $\stackrel{r}{T} M$ into $\stackrel{r}{T}^{r}$ of maximal rank.

Proof. We shall prove only for the case $r=2$, since the proof for $r \geqq 3$ is similar. Let $p_{0} \in M$ and put $q_{0}=\Phi\left(p_{0}\right)$. We take a coordinate 
neighborhood $U$ (resp. $V$ ) of $p_{0}$ (resp. $q_{0}$ ) with coordinate system $\left\{x_{1}, \cdots, x_{n}\right\}$ (resp. $\left\{y_{1}, \cdots, y_{m}\right\}$ ) such that $\Phi(U) \subset V$. Then, $\stackrel{2}{T} U$ (resp. $\stackrel{2}{T} V$ ) has the induced coordinate system $\left\{x_{i}, \dot{x}_{i}, \ddot{x}_{i} \mid i=1,2, \cdots, n\right\}$ (resp. $\left\{y_{j}, \dot{y}_{j}, \ddot{y}_{j} \mid j=1,2\right.$, $\cdots, m\})$. Put $F_{i}\left(x_{1}, \cdots, x_{n}\right)=y_{i}(\Phi(x))$ for $x \in U$. Take an element $[\varphi]_{2} \in \stackrel{2}{T}(U)$ with coordinates $\left\{x_{i}, \dot{x}_{i}\right.$, $\left.\ddot{x}_{i}\right\}$, then $x_{i}(\varphi(t))=x_{i}+\dot{x}_{i} t+\ddot{x}_{i} t^{2}+\varepsilon_{i}(t)$, where $\left[d^{2} \varepsilon_{i} / d t^{2}\right]_{0}=0$. Hence, we have $y_{i} \Phi\left(x_{1}(\varphi(t)), \cdots, x_{n}(\varphi(t))\right)=F_{i}\left(x_{1}, \cdots, x_{n}\right)$ $+\sum_{j} \frac{\partial F_{i}}{\partial x_{j}} \dot{x}_{j} t+\frac{1}{2}\left(\sum_{j, k} \frac{\partial^{2} F_{i}}{\partial x_{j} \partial x_{k}} \dot{x}_{k} \dot{x}_{j}+2 \sum_{j} \frac{\partial F_{i}}{\partial x_{j}} \ddot{x}_{j}\right) t^{2}+\eta_{i}(t)$, where $\left[d^{2} \eta_{i} / d t^{2}\right]_{0}$ $=0$. Therefore, $(\stackrel{2}{T} \Phi)\left([\varphi]_{2}\right)=[\Phi \circ \varphi]_{2}$ has the following coordinates:

$$
\left\{\begin{array}{l}
y_{i}=F_{i}(x), \quad \dot{y}_{i} \sum_{j} \frac{\partial F_{i}}{\partial x_{j}} \dot{x}_{j}, \\
\ddot{y}_{i}=\frac{1}{2} \sum_{j, k} \frac{\partial^{2} F_{i}}{\partial x_{j} \partial x_{k}} \dot{x}_{j} \dot{x}_{k}+\sum_{j} \frac{\partial F_{i}}{\partial x_{j}} \ddot{x}_{j}
\end{array}\right.
$$

Hence, the map $\stackrel{2}{T \Phi}$ has the Jacobian matrix $J$ with respect the coordinate systems $\left\{\stackrel{(\nu)}{x_{i}} \mid i=1, \cdots, n ; \nu=0,1,2\right\}$ and $\left\{y_{k} \mid k=1, \cdots, m ; \nu=0,1,2\right\}$ as follows:

$$
J=\left(\begin{array}{ccc}
\left(\frac{\partial F_{i}}{\partial x_{k}}\right) & 0 & 0 \\
\left(J_{k}^{i}\right) & \left(\frac{\partial F_{i}}{\partial x_{k}}\right) & 0 \\
\left(\ddot{J}_{k}^{i}\right) & \left(\dot{J}_{k}^{i}\right) & \left(\frac{\partial F_{i}}{\partial x_{k}}\right)
\end{array}\right)
$$

where $\dot{J}_{k}^{i}=\sum_{j} \frac{\partial^{2} F_{i}}{\partial x_{j} \partial x_{k}} \dot{x}_{j}$ and $\ddot{J}_{k}^{i}=\frac{1}{2} \sum_{j, l} \frac{\partial^{3} F_{i}}{\partial x_{j} \partial x_{k} \partial x_{l}} \dot{x}_{j} \cdot \dot{x}_{l}+\sum_{j} \frac{\partial^{2} F_{i}}{\partial x_{j} \partial x_{k}} \ddot{x}_{j}$

Since the Jacobian matrix of $\Phi$ is $\left(\frac{\partial F_{i}}{\partial x_{k}}\right)$, which has the maximal rank, $J$ has also the maximal rank.

Corollary 1.9. Let $\Phi$ be a regular map of $M$ into $N$, namely the differential $T \Phi$ is an injective map of $T_{p}(M)$ into $T_{\Phi(p)}(N)$ for every point $p \in M$. Then, $\stackrel{r}{T} \Phi$ is also a regular map of $\stackrel{r}{T} M$ into $T N$.

Remark 1. 10. We see that if $\Phi$ is a regular injective map, then $\stackrel{r}{T} \Phi$ is also a regular injective map. 


\section{§2. Tangent groups of order $r$.}

Let $G$ be a Lie group with group multiplication $\mu: G \times G \rightarrow G$ and with the unit element $e$.

THeOREM 2. 1. $\stackrel{r}{T} G$ is a Lie group with group multiplication $\stackrel{r}{T} \mu . \quad$ The group $G$ is a closed subgroup of $\stackrel{r}{T} G$ and $\stackrel{r}{T}_{e}(G)$ is a closed normal subgroup of $\stackrel{r}{T} G$ such that

$$
\stackrel{r}{T} G=G \cdot \stackrel{r}{T}_{e}(G),
$$

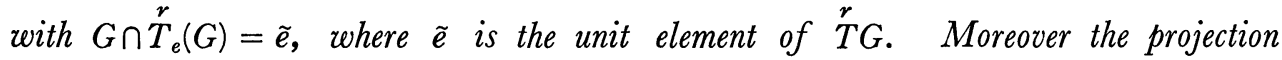
$\stackrel{r}{\pi} \stackrel{r}{T} G \rightarrow G$ is a homomorphism. (cf. [3] for $r=1$ )

Proof. For any two elements $\varphi, \psi \in S(G)$ (cf. \$1), we define $\varphi \cdot \psi \in S(G)$ by $(\varphi \cdot \psi)(t)=\varphi(t) \cdot \psi(t)$ for $t \in R$. Then, we have $(\stackrel{r}{T} \mu)\left([\varphi]_{r},[\psi]_{r}\right)=(\stackrel{r}{T} \mu)$ $\left([(\varphi, \psi)]_{r}\right)=[\mu \circ(\varphi, \psi)]_{r}=[\varphi \cdot \psi]_{r}$ and hence we get

$$
(\stackrel{r}{T} \mu)\left([\varphi]_{r},[\psi]_{r}\right)=[\varphi \cdot \psi]_{r} .
$$

Since $(\varphi \cdot \psi) \cdot \eta=\varphi \cdot(\psi \cdot \eta)$ for any $\varphi, \psi, \eta \in S(G)$, we see that the multiplication $\stackrel{r}{T} \mu$ is associative. Define $\gamma_{e} \in S(G)$ by $\gamma_{e}(t)=e$ for $t \in R$ and put $\tilde{e}=\left[\gamma_{e}\right]_{r}$. Clearly $\tilde{e}$ is the unit element with respect to $T_{\mu}^{r}$. For $\varphi \in S(G)$, we define $\varphi^{-1} \in S(G)$ by $\varphi^{-1}(t)=(\varphi(t))^{-1}$ for $t \in R$. Then $\stackrel{r}{T} \mu\left([\varphi]_{r},\left[\varphi^{-1}\right]_{r}\right)=$ $\left[\varphi \cdot \varphi^{-1}\right]_{r}=\left[\gamma_{e}\right]_{r}=\tilde{e}$ and hence $\left[\varphi^{-1}\right]_{r}$ is the inverse element of $[\varphi]_{r}$. Now, $\left[\varphi^{-1}\right]_{r}=(\stackrel{r}{T} \iota)[\varphi]_{r}$, where $\iota: G \rightarrow G$ is the map $x \rightarrow x^{-1}$ for $x \in G$. Since $\stackrel{r}{T_{\iota}}$ is a differentiable map of $\stackrel{r}{T} G$ into itself, we have proved that $\stackrel{r}{T} G$ is a $\mathrm{Lie}$ group with group multiplication $\stackrel{r}{T} \mu$. Next, since $G=\left\{\left[\gamma_{a}\right]_{r} \mid a \in G\right\}$, where $\gamma_{a}(t)=a$ for $t \in R$, it follows that $G$ is a closed subgroup of $\stackrel{r}{T} G$. Similarly we see that $\stackrel{r}{T_{e}} G$ is a closed normal subgroup of $\stackrel{r}{T} G$. Next, any $[\varphi]_{r} \in \stackrel{r}{T} G$ can be written as $[\varphi]_{r}=\left[\gamma_{a}\right]_{r} \cdot\left[\gamma_{a^{-1}} \cdot \varphi\right]_{r}$, where $a=\varphi(0)$ and so $\left[\gamma_{a^{-1}} \cdot \varphi\right]_{r} \in \stackrel{r}{T_{e} G}$. The equality $G \cap \stackrel{r}{T}_{e} G=\tilde{e}$ is also clear. Finally the projection $\underset{r}{r}$ is a homomorphism since (2.1) holds.

Q.E.D.

Definition 2. 2. The Lie group $T G$ with group multiplication $\stackrel{r}{T} \mu$ will be called the tangent group to $G$ of order $r$. 
Proposition 2. 3. Let $\Phi$ be a homomorphism of a Lie group $G$ into a Lie group $G^{\prime}$. Then $\stackrel{r}{T} \Phi$ is also a homomorphism of the tangent group $\stackrel{r}{T} G$ of order $r$ into $\stackrel{r}{T} G^{\prime}$.

Proof. Let $\mu^{\prime}$ be the group multiplication of $G$. Since $\Phi$ is a homomorphism, we have $\Phi \circ \mu=\mu^{\prime} \circ(\Phi \times \Phi)$. By Proposition 1.6 we have $\stackrel{r}{T} \Phi \circ \stackrel{r}{T} \mu$ $=\stackrel{r}{T} \mu^{\prime} \circ(\stackrel{r}{T} \Phi \times \stackrel{r}{T} \Phi)$, which means that $\stackrel{r}{T} \Phi$ is a homomorphism of $\stackrel{r}{T} G$ into $\stackrel{r}{T} G^{\prime}$.

Proposition 2. 4. The projection $\pi_{s}^{r}: \stackrel{r}{T} G \rightarrow \stackrel{r}{T} G$ for $r>s$ is $s$ a homomorphism of tangent groups.

Proof. Clear from the equality (2. 1).

Proposition 2. 5. If $G$ is a Lie subgroup of $G^{\prime}$, then $\stackrel{r}{T}(G)$ is also a Lie subgroup of $\stackrel{r}{T}\left(G^{\prime}\right)$.

Proof. Let $\Phi: G \rightarrow G^{\prime}$ be the injection map. Then $\Phi$ is a regular map. By Remark 1.10 and Proposition 2.3, $\stackrel{r}{T \Phi}$ is a regular homomorphism of $T G$ into $\stackrel{r}{T} G^{\prime}$. Let $[\varphi]_{r}$ be an element of $\stackrel{r}{T} G$ such that $\stackrel{r}{(T \Phi)}\left([\varphi]_{r}\right)=\tilde{e}^{\prime}$ is the unit element of $\stackrel{r}{T} G^{\prime}$. Then $[\Phi \circ \varphi]_{r}=\left[\gamma_{e}^{\prime}\right]_{r}$, where $\gamma_{e}^{\prime}: R \rightarrow G^{\prime}$ is defined by $\gamma_{e}^{\prime}(t)=e$ for $t \in R$, $e$ being the unit element of $G$. We see that $\varphi(0)=e$ and that $[\varphi]_{r}=\left[\gamma_{e}\right]=\tilde{e}$. Hence $\stackrel{r}{T} \Phi$ is a regular injective homomorphism, which means that $\stackrel{r}{T}_{G}$ is a Lie subgroup of $\stackrel{r}{T} G^{\prime}$.

Q.E.D.

\section{\$3. Tangent operations of order $\boldsymbol{r}$.}

Let $G$ be a Lie group operating on a manifold $M$ differentiably. We denote by $\rho: G \times M \rightarrow M$ the operation map of $G$ on $M$.

Proposition 3. 1. The tangent group $\stackrel{r}{T} G$ to $G$ of order $r$ operates on the tangent bundle $\stackrel{r}{T} M$ of order $r$ by the operation map $\stackrel{r}{T} \rho$ (for the tangent group $T G$, see [3]).

Proof. Since $\rho$ is the operation map of $G$ on $M$, we have $\rho \circ\left(\mu \times 1_{M}\right)$ $=\rho \circ\left(1_{G} \times \rho\right)$. By Proposition 1. 6 we have $(\stackrel{r}{T} \rho) \circ\left(\stackrel{r}{T} \mu \times 1_{T_{T}}\right)=\stackrel{r}{T} \rho \circ\left(1_{r G}^{r} \times \stackrel{r}{T} \rho\right)$, which means that $\tilde{a} \cdot(\tilde{b} \cdot \tilde{x})=(\tilde{a} \cdot \tilde{b}) \cdot \tilde{x}$ for $\tilde{a}, \tilde{b} \in \stackrel{r}{T}(G)$ and $\tilde{x} \in \stackrel{r}{T} M$, where we 
have put $\tilde{a} \cdot \tilde{x}=(\stackrel{r}{T} \rho)(\tilde{a}, \tilde{x})$. Let $\gamma_{e}: R \rightarrow G$ be the constant map: $\gamma_{e}(t)=e$ for $t \in R$. Then, for any $[\varphi]_{r} \in \stackrel{r}{T} M$ we have $\left.\stackrel{r}{T} \rho\left(\left[\gamma_{e}\right],[\varphi]_{r}\right)=\stackrel{r}{T} \rho\left(\left[\gamma_{e}, \varphi\right)\right]_{r}\right)=$ $\left.\left[\rho \circ\left(\gamma_{e}, \varphi\right)\right]_{r}=\left[\gamma_{e}, \varphi\right)\right]_{r}=[\varphi]_{r}$, which means that the unit element $\tilde{e}=\left[\gamma_{e}\right]_{r}$ of $\stackrel{r}{T} G$ operates on $\stackrel{r}{T} M$ as the identity map. Hence we have proved that $\stackrel{r}{T} G$ operates on $\stackrel{r}{T} M$ by $\stackrel{r}{T} \rho$.

Q.E.D.

Definition 3. 2. The operation map $\stackrel{r}{T} \rho$ in Proposition 3.1 will be called the tangent operation to $\rho$ of order $r$.

Proposition 3. 3. If a Lie group $G$ operates on $M$ effectively (i.e. $a \cdot x=x$ for all $x \in M$ implies $a=e$ ), then $\stackrel{2}{T} G$ operaties on $\stackrel{2}{T} M$ effectively by the tangent operation of order 2 .

Proof. For $\varphi \in S(G)$ and $\psi \in S(M)$ we define $\varphi \cdot \psi \in S(M)$ by $(\varphi \cdot \psi)(t)=$ $\varphi(t) \cdot \psi(t)$ for $t \in R$. Suppose $\varphi \cdot \psi \widetilde{\widetilde{\tau}^{2}} \psi$ for every $\phi \in S(M)$. We have to show that $\varphi \widetilde{{ }_{2}} \gamma_{e}$, where $\gamma_{e} \in S(G)$ is defined by $\gamma_{e}(t)=e$. First, since $\varphi(0) \cdot \psi(0)=\psi(0)$ for any $\psi \in S(M)$, we see that $\varphi(0) \cdot x=x$ for any $x \in M$, whence $\varphi(0)=e$ since $G$ operates effectively on $M$. Next take a point $p_{0} \in M$ and fix it. We take a coordinate neighborhhod $U$ (resp. $V$ ) of $p_{0}$ (resp. of $e$ ) in $M$ (resp. in $G$ ) with coordinate system $\left\{x_{1}, \cdots, x_{n}\right\}$ (resp. $\left\{z_{1}, \cdots, z_{N}\right\}$ ) such that $x_{i}\left(p_{0}\right)=0$ for $i=1,2, \cdots, n\left(\operatorname{resp} . z_{l}(e)=0\right.$ for $\left.l=1,2, \cdots, N\right)$. Define the functions $F_{i}(i=1, \cdots, n)$ by

$$
F_{i}\left(z_{1}, \cdots, z_{N} ; x_{1}, \cdots, x_{n}\right)=x_{i}(\rho(z, x)) .
$$

Let $\left\{x_{i}^{(\nu)} \mid i=1, \cdots, n ; \nu=0,1,2\right\}$ (resp. $\left.\left\{\stackrel{(\nu)}{z_{l}} \mid l=1, \cdots, N ; \nu=0,1,2\right\}\right)$ be the induced coordinate system on $\stackrel{r}{T}(U)$ (resp. $\stackrel{r}{T}(V))$. If $\stackrel{(0)}{x_{i}}\left([\psi]_{2}\right)=x_{i}, \stackrel{(1)}{x_{i}}\left([\psi]_{2}\right)=\dot{x}_{i}$, $\stackrel{(2)}{x_{i}}\left([\psi]_{2}\right)=\ddot{x}_{i}$, we see that

$$
\psi(t)=\left(\cdots, x_{i}+\dot{x}_{i}+\ddot{x}_{i} t^{2}+\varepsilon_{i}(t), \cdots\right) \in U
$$

for small $|t|$, where $\left[d^{2} \varepsilon_{i} / d t^{2}\right]_{0}=0$ for $i=1, \cdots, n$. Similarly we see that

$$
\varphi(t)=\left(\cdots, \dot{z}_{l} t+\ddot{z}_{l} t+\eta_{l}(t), \cdots\right) \in V
$$

for small $|t|$, where $\left[d^{2} \eta_{l} / d t^{2}\right]_{0}=0$ for $l=1, \cdots, N$. We have the relations $x_{i} \circ(\varphi \cdot \psi) \underset{r}{\sim} x_{i} \circ \psi(i=1,2, \cdots, n)$ for every $\phi \in S(M)$. To simplify the notations we define the functions $f_{i}(t)$ for $i=1, \cdots, n$ by

$$
f_{i}(t)=F_{i}\left(\cdots, \varphi_{l}(t), \cdots ; \cdots, \phi_{i}(t), \cdots\right)
$$


and we define the variables $\stackrel{(\nu)}{y_{k}}$ for $\kappa=1,2, \cdots N+n ; \nu=0,1, \cdots, r$ by $\stackrel{(\nu)}{y_{\kappa}}=\nu ! \cdot{ }^{(\nu)}$ for $\kappa=1,2, \cdots, N$ and $\stackrel{(\nu)}{y}_{k}=\nu ! \cdot{ }^{(\nu)} x_{\kappa-N}$ for $\kappa=N+1, \cdots, N+n$. By means of these notations we have the following equalities

$$
\begin{aligned}
& \frac{d f_{i}}{d t}=\sum_{\kappa=1}^{N+n} \frac{\partial F_{i}}{\partial y_{\kappa}}\left(\dot{y}_{\kappa}+\ddot{y}_{\kappa} t+\varepsilon_{1}^{\kappa}(t)\right) \\
& \frac{d^{2} f_{i}}{d t^{2}}=\sum_{\kappa} \frac{\partial F_{i}}{\partial y_{\kappa}}\left(\ddot{y}_{\kappa}+\stackrel{(3)}{y}_{\kappa} t+\varepsilon_{2}^{\kappa}(t)\right) \\
& +\sum_{\kappa, \lambda} \frac{\partial^{2} F_{i}}{\partial y_{\kappa} \partial y_{\lambda}}\left(\dot{y}_{\kappa}+\ddot{y}_{\kappa} t+\varepsilon_{1}^{\kappa}(t)\right) \cdot\left(\dot{y}_{\lambda}+\ddot{y}_{\lambda} t+\varepsilon_{1}^{\lambda}(t)\right) \text {, }
\end{aligned}
$$

where $\left[d \varepsilon_{k}^{\kappa} / d t\right]_{0}=0$ for $k=1,2$. Since $f_{i}(t)=\left(x_{i} \circ(\varphi \cdot \psi)\right)(t)$ and since $x_{i} \circ(\varphi \cdot \psi) \sim x_{i} \circ \psi$ we obtain the following relations:

$$
\begin{aligned}
& \sum_{l=1}^{N}\left[\frac{\partial F_{i}}{\partial z}\right]_{(0, x)} \dot{z}_{l}+\sum_{j=1}^{n}\left[\frac{\partial F_{i}}{\partial x_{j}}\right]_{(0, x)} \dot{x}_{j}=\dot{x}_{i}, \\
& 2 \sum_{l}\left[\frac{\partial F_{i}}{\partial z_{l}}\right]_{(0, x)} \ddot{z}_{l}+2 \sum_{j}\left[\frac{\partial F_{i}}{\partial x_{j}}\right]_{(0, x)} \ddot{x}_{j} \\
+ & \sum_{l, m=1}^{N}\left[\frac{\partial^{2} F_{i}}{\partial z_{l} \partial z_{m}}\right]_{(0, x)} \dot{z}_{l} \cdot \dot{z}_{m}+2 \sum_{l=1}^{N} \sum_{j=1}^{n}\left[\frac{\partial^{2} F_{i}}{\partial z_{l} \partial x_{j}}\right]_{(0, x)} \dot{z}_{l} \dot{x}_{j} \\
+ & \sum_{j, k=1}^{n}\left[\frac{\partial F_{i}}{\partial x_{j} \partial x_{k}}\right]_{(0, x)} \dot{x}_{j} \dot{x}_{k}=\ddot{x}_{i}
\end{aligned}
$$

for $i=1,2, \cdots, n$ and for every $\left(x_{i}, \dot{x}_{i}, \ddot{x}_{i}\right) \in U$. Now, since $e \cdot x=x$ for any $x \in M$, we have

$$
F_{i}\left(0, \cdots, 0 ; x_{1}, \cdots, x_{n}\right)=x_{i}
$$

for $i=1,2, \cdots, n$. Therefore, we get $\left[\frac{\partial F_{i}}{\partial x_{j}}\right]_{(0, x)}=\delta_{j}^{i}$ for $i, j=1,2, \cdots, n$. Finally, we obtain. from (3.3), (3.4) the following relations:

$$
\begin{aligned}
& \sum_{l=1}^{N}\left[\frac{\partial F_{i}}{\partial z}\right]_{(0, x)} \dot{z}_{l}=0 . \\
& 2 \sum\left[\frac{\partial F_{i}}{\partial z_{l}}\right]_{(0, x)} \ddot{z}_{l}+\sum_{l, m}\left[\frac{\partial^{2} F_{i}}{\partial z_{l} \partial z_{m}}\right]_{(0, x)} \dot{z}_{l} \dot{z}_{m} \\
+ & 2 \sum_{l, j}\left[\frac{\partial^{2} F_{i}}{\partial z_{l} \partial x_{j}}\right]_{(0, x)} \dot{z}_{l} \cdot \dot{x}_{j}=0
\end{aligned}
$$

for every $\left(x_{1}, \cdots, x_{n}\right) \in U$ and $i=1, \cdots, n$.

Now, we shall prove the following 
Lemma 3. 4. Let $a_{1}, \cdots, a_{N} \in R$. Suppose $\sum_{l=1}^{N} a_{l}\left[\frac{\partial F_{i}}{\partial z_{l}}\right]_{(0, x)}=0$ holds for every $\left(x_{1}, \cdots, x_{n}\right) \in U$ and for $i=1,2, \cdots, n$, where $U$ is an arbitrary coordinate neighborhood in $M$. Then $a_{l}=0$ for $l=1,2, \cdots, N$.

By virtue of this lemma, we see from (3.5) that $\dot{z}_{l}=0$ for $l=1,2, \cdots$, $N$ and then from (3.6) it follows that $\ddot{z}_{l}=0$ for $l=1,2, \cdots, N$, which proves that $\varphi \underset{2}{\sim} \gamma_{e}$ and thus the proposition will be proved.

Proof of Lemma 3. 4. Suppose $a_{l} \neq 0$ for some $l$. Let $g$ be the Lie algebra of $G$. By taking a linear transformation of the coordinates $\left\{z_{1}, \cdots\right.$, $\left.z_{N}\right\}$, if necessary, we can suppose that $\left[\partial F_{i} / \partial z_{1}\right]_{(0, x)}=0$ for any $x \in U$ and that $z_{i}\left(\exp \sum_{j=1}^{N} t_{j} X_{j}\right)=t_{i}$ for $i=1,2, \cdots, N$, where $\left\{X_{1}, \cdots, X_{N}\right\}$ is a base of $g$. Now let $\tilde{X}_{1}$ be the vector field on $M$ induced by the one-parameter group $\exp t X_{1}$. For any point $x \in U$, we have $\left(\tilde{X}_{1}\right)_{x}=0$, since $\left(\tilde{X}_{1}\right)_{x} \cdot x_{i}=$ $\left[d x_{i}\left(\left(\exp t X_{1}\right) \cdot x\right) / d t\right]_{0}=\left[d F_{i}(t, 0, \cdots, 0 ; x) / d t\right]_{0}=\left[\partial F_{i} / \partial z_{1}\right]_{(0, x)}=0$ for $i=1,2$, $\cdots, n$. Since $U$ and $x$ are arbitrary, we see that $\tilde{X}_{1}=0$ on $M$ and that $\exp t X_{1}$ operates trivially on $M$. It follows that $\exp t X_{1}=e$ for any $t \in R$ and hence $X_{1}=0$, which is a contradiction. Thus Lemma 3.4 is proved and hence the proof of Proposition 3. 3 is complete.

Q.E.D.

More generally, we can prove the following

TH: OREM 3. 5. If a Lie group $G$ operates on $M$ effectively, then $\stackrel{r}{T} G$ operates on $\stackrel{r}{T} M$ effectively by tangent operation of order $r$ for any poistive integer $r$.

Proof. Using the notations of the proof of Proposition 3. 3, especially the notations of (3. 1), we define $\varphi_{\alpha}(t)$ by $\varphi_{\alpha}(t)=\dot{y}_{\alpha}+\ddot{y}_{\alpha} t+\varepsilon_{1}^{\alpha}(t)$ for $\alpha=1,2$, $\cdots, N+n$. Then the equality (3.2) can be written as follows:

$$
\frac{d^{2} f_{i}}{d t^{2}}=\Sigma \frac{\partial F_{i}}{\partial y_{\alpha}} \cdot \varphi_{\alpha}+\Sigma \frac{\partial^{2} F_{i}}{\partial y_{\alpha} \partial y_{\beta}} \varphi_{\alpha} \varphi_{\beta}
$$

By differentiating (3. 7), we obtain the following

$$
\begin{aligned}
\frac{d^{3} f_{i}}{d t^{3}} & =\sum \frac{\partial^{3} F_{i}}{\partial y_{\alpha} \partial y_{\beta} \partial y_{\gamma}} \varphi_{\alpha} \varphi_{\beta} \varphi_{\gamma} \\
& +3 \sum \frac{\partial^{2} F_{i}}{\partial y_{\alpha} \partial y_{\beta}} \varphi_{\alpha}^{\prime} \varphi_{\beta}+\sum \frac{\partial F_{i}}{\partial y_{\alpha}} \varphi_{a .}^{\prime \prime}
\end{aligned}
$$

In general, by induction on $\nu=1,2, \cdots$, we obtain the following equality 


$$
\begin{aligned}
\frac{d^{\nu} f_{i}}{d t^{\nu}} & =\sum \frac{\partial^{\nu} F_{i}}{\partial y_{\alpha_{1}} \cdots \partial y_{\alpha_{\nu}}} \varphi_{\alpha_{1}} \cdots \varphi_{\alpha_{\nu}} \\
& +c_{1}^{(\nu)} \sum \frac{\partial^{\nu-1} F_{i}}{\partial y_{\alpha_{1}} \cdots \partial y_{\alpha_{\nu-1}}} \varphi_{\alpha_{1}}^{\prime} \varphi_{\alpha_{2}} \cdots \varphi_{\alpha_{\nu-1}} \\
& +c_{1,1}^{(\nu)} \sum \frac{\partial^{\nu-2} F_{i}}{\partial y_{\alpha_{1}} \cdots \partial y_{\alpha_{\nu-2}}} \varphi_{\alpha_{1}}^{\prime} \varphi_{\alpha_{2}}^{\prime} \varphi_{\alpha_{3}} \cdots \varphi_{\alpha_{\nu-2}} \\
& +c_{2}^{(\nu)} \sum \frac{\partial^{\nu-2} F_{i}}{\partial y_{\alpha_{1}} \cdots \partial y_{\alpha_{\nu}-2}} \varphi_{\alpha_{1}}^{\prime \prime} \varphi_{\alpha_{2}} \cdots \varphi_{\alpha_{\nu-2}} \\
& +\cdots+c_{\nu-2}^{(\nu)} \sum \frac{\partial^{2} F_{i}}{\partial y_{\alpha_{1}} \partial y_{\alpha_{2}}} \varphi_{1}^{(\nu-2)} \varphi_{\alpha_{2}}+\sum \frac{\partial F_{i}}{\partial y_{\alpha}} \varphi_{\alpha}^{(\nu-1)},
\end{aligned}
$$

where $c_{\mu_{1} \cdots \mu_{e}}^{(\nu)}$ are some positi e integer for $\sum_{i=1}^{s} \mu_{i}=1,2, \cdots, \nu-2$ and for any $\nu=1,2, \cdots$.

Suppose $(\varphi \cdot \psi) \widetilde{r} \psi$ for every $\psi \in S(M)$ as in the proof of Proposition 3. 3. By using (3.9) and Lemma 3. 4 repeatedly we can show, by induction on $\nu$, that $z_{l}=0$ for any $l=1,2, \cdots, N$ and $\nu=0,1, \cdots, r$, which proves that $\varphi \underset{r}{\sim} r_{e}$.

Q.E.D.

\section{\$4. Tangent bundle to $R^{n}$ of order $r$.}

Let $R^{n}$ be the real euclidean space of dimension $n$. For any two $r$ tangents $[\varphi]_{r},[\psi]_{r}$ to $R^{n}$, we define their sum by: $[\varphi]_{r}+[\psi]_{r}=[\varphi+\psi]_{r}$, where $(\varphi+\psi)(t)=\varphi(t)+\psi(t)$ for $t \in R$. For any $c \in R$ we define the scalar multiplication of $[\varphi]_{r}$ by $c$ as follows: $c \cdot[\varphi]_{r}=[c \cdot \varphi]_{r}$, where $(c \cdot \varphi)(t)=c \cdot \varphi(t)$ for $t \in R$. Clearly $[\varphi]_{r}+[\psi]_{r}$ and $c \cdot[\varphi]_{r}$ are well-defined.

THEOREM 4.1. By the above sum and scalar multiplication the tangent bundle $\stackrel{r}{T} R_{n}$ to $R^{n}$ of order $r$ is a real vector space of dimension $n(r+1)$.

Proof. Straightforward verification.

Proposition 4.2. Let $V \oplus W$ be a direct sum of vector subspaces $V$ and $W$, then $\stackrel{r}{T} V$ and $\stackrel{r}{T} W$ are identified with vector subspaces of $T(V \oplus W)$ and we have

$$
\stackrel{r}{T}(V \oplus W)=\stackrel{r}{T} V \oplus \stackrel{r}{T} W(\text { direct } \text { sum })
$$

Remark 4. 3. Let $\left\{x_{1}, \cdots, x\right\}$ be the natural coordinate system on $R^{n}$ and let $\left\{x_{i} \mid i=1, \cdots, n ; \nu\right\}$ be the induced coo dinate system on $\stackrel{r}{T} R^{n}$. 
Then the sum and scalar multiplication in $\stackrel{r}{T} R^{n}$ in Theorem 4.1 are as follows:

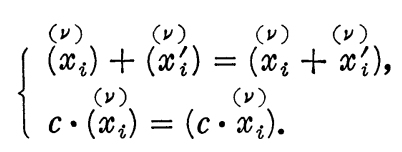

§5. Imbedding of $\stackrel{r}{T} G L(n)$ into $G L(n(r+1))$.

Let $\rho: G L(n) \times R^{n} \rightarrow R^{n}$ be the usual operation of the general linear group $G L(n)$ on $R^{n}$. By Proposition 3.1, the tangent group $\stackrel{r}{T} G L(n)$ to $G L(n)$ of order $r$ operates on $\stackrel{r}{T} R^{n}$ by the tangent operation $\stackrel{r}{T} \rho$ to $\rho$ of order $r$. Now, by Theorem 4. 1, $\stackrel{r}{T} R^{n}$ is a vector space of dimension $n(r+1)$. We shall prove the following

TheOREM 5. 1. The tangent group $\stackrel{r}{T} G L(n)$ to $G L(n)$ of order $r$ operates on $\stackrel{r}{T} R^{n}$ effectively as a linear group.

Proof. Since $\rho$ is effective, we see that $\stackrel{r}{T} \rho$ is effective by Theorem 3. 5. For any $\eta \in S(G L(n))$ and $\varphi \in S\left(R^{n}\right)$, we define $\eta \cdot \varphi \in S\left(R^{n}\right)$ by the equality $(\eta \cdot \varphi)(t)=\eta(t) \cdot(t)=\rho(\eta(t), \varphi(t))$ for $t \in R$. We put $[\eta]_{r} \cdot[\varphi]_{r}=\stackrel{r}{T} \rho\left([\eta]_{r},[\varphi]_{r}\right)$. Then we have $[\eta]_{r} \cdot[\varphi]_{r}=[\eta \cdot \varphi]_{r}$. Take an element $[\psi]_{r}$ of $\stackrel{r}{T}\left(R^{n}\right)$ and $c \in R$. Then we calculate as follows: $[\eta]_{r}\left([\varphi]_{r}+[\psi]_{r}\right)=[\eta]_{r} \cdot[\varphi+\psi]_{r}=[\eta \cdot(\varphi+\psi)]_{r}$ $=[\eta \cdot \varphi+\eta \cdot \psi]_{r}=[\eta \cdot \varphi]_{r}+[\eta \cdot \psi]_{r}=[\eta]_{r} \cdot[\varphi]_{r}+[\eta]_{r} \cdot[\psi]_{r}$. Similarly, we have $[\eta]_{r}\left(c \cdot[\varphi]_{r}\right)=[\eta]_{r}[c \cdot \varphi]_{r}=[\eta \cdot(c \varphi)]_{r}=[c \cdot(\eta \cdot \varphi)]_{r}=c[\eta \cdot \varphi]_{r}=c\left([\eta]_{r} \cdot[\varphi]_{r}\right)$. Thus we have proved that $[\eta]_{r}$ operates on $\stackrel{r}{T} R^{n}$ as a linear transformation.

$$
\text { Q.E.D. }
$$

Definition 5.2. Let $\left\{x_{1}, \cdots, x_{n}\right\}$ be the natural coordinate system on $R^{n}$ and let $\left\{x_{i} \mid i=1, \cdots, n ; \nu=0,1, \cdots, r\right\}$ be the induced coordinate system on $\stackrel{r}{T} R^{n}$. Using these coordinates, Theorem 5.1 shows that there is a canonical injective homomorphism $j_{n}^{(r)}$ of $T G L(n)$ into $G L(n(r+1))$.

Let $\left(y_{j}^{i}\right) \in G L(n)$. Then $\stackrel{r}{T} G L(n)$ has the induced coordin te system $\left\{y_{j}^{i} \mid i, j=1, \cdots, n ; \nu=0,1, \cdots, r\right\}$. We denote by $Y_{\nu}$ the $n \times n$-matrix

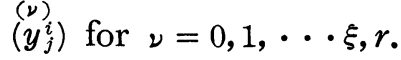


Proposition 5. 3. The homomorphism $j_{n}^{(r)}$ is given by the following equality:

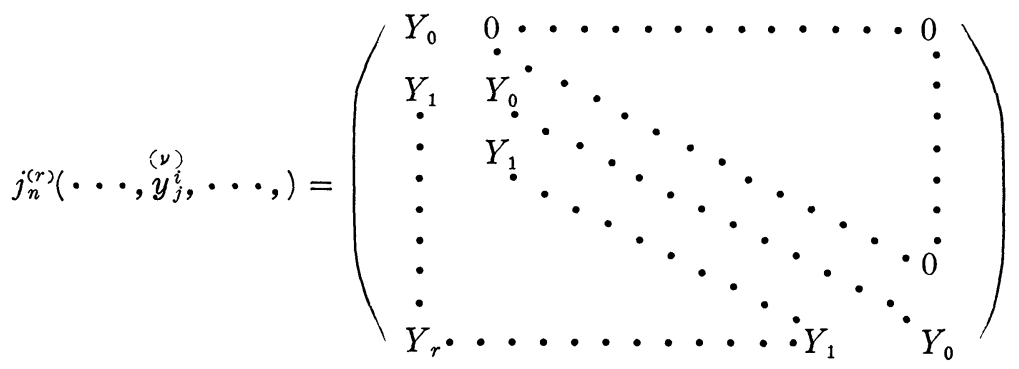

Proof. We shall prove the proposition only for the case $r=2$, since the proof for the case $r \geqq 3$ is similar. Let $[\varphi]_{2} \in \stackrel{2}{T} G L(n)$ be such that $[\varphi]_{2}$

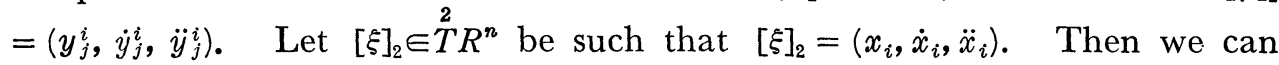
assume that

$$
\left\{\begin{array}{l}
\varphi(t)=\left(y_{j}^{i}+\dot{y}_{j}^{j} t+\ddot{y}_{j}^{i} t^{2}\right), \\
\xi(t)=\left(x_{i}+\dot{x}_{i} t+\ddot{x}_{i} t^{2}\right)
\end{array}\right.
$$

for $t \in R$. From (5. 1) it follows that $(\varphi \cdot \xi)(t)=\varphi(t) \cdot \xi(t)=\left(\sum_{i}\left(y_{j}^{i}+\dot{y}_{j}^{i} t+\right.\right.$ $\left.\left.\ddot{y}_{j}^{i} t^{2}\right)\left(x_{i}+\dot{x}_{i} t+\ddot{x}_{i} t^{2}\right)\right)=\left(\sum_{i} y_{j}^{i} x_{i}+\sum\left(\dot{y}_{j}^{i} x_{i}+y_{j}^{i} \dot{x}_{i}\right) t+\sum_{i}\left(y_{j}^{i} \ddot{x}_{i}+\dot{y}_{j}^{i} \dot{x}_{i}+\ddot{y}_{j}^{i} x_{i}\right) t^{2}\right.$ $\left.+\sum_{i}\left(\dot{y}_{j}^{i} \ddot{x}_{i}+\ddot{y}_{j}^{i} \dot{x}_{i}\right) t^{3}+\sum_{i} \ddot{y}_{j}^{i} \ddot{x}_{i} t^{4}\right)$. Therefore, we get $[\varphi]_{2}[\xi]_{2}=[\varphi \cdot \xi]_{2}=\left(\sum_{i} y_{j}^{i} x_{i}\right.$, $\left.\sum_{i}\left(\dot{y}_{j}^{i} x_{i}+y_{j}^{i} \dot{x}_{i}\right), \sum_{i}\left(y_{j}^{i} \ddot{x}_{i}+\dot{y}_{j}^{i}+\dot{x}_{i}+\ddot{y}_{j}^{i} x_{i}\right)\right)$,

and hence we obtain

$$
j_{n}^{(2)}\left([\varphi]_{2}\right)=\left(\begin{array}{ccc}
y_{j}^{i} & 0 & 0 \\
\dot{y}_{j}^{i} & y_{j}^{i} & 0 \\
\ddot{y}_{j}^{i} & \dot{y}_{j}^{i} & y_{j}^{i}
\end{array}\right)
$$

which proves the proposition.

Q.E.D.

\section{§6. Tangential fibre bundle of order $r$.}

Let $E(M, \pi, F, G)$ be a fibre bundle with bundle space $E$, base $M$, projection $\pi$, fibre $F$ and structure group $G$. We shall prove the following

Proposition 6. 1. $\stackrel{r}{T} E(\stackrel{r}{T} M, \stackrel{r}{T} \pi, \stackrel{r}{T} F, \stackrel{r}{T} G)$ is a fibre bundle with bundle space $\stackrel{r}{T E}$, base $\stackrel{r}{T} M$, projection $\stackrel{r}{T} \pi$, fibre $\stackrel{r}{T} F$ and structure gro $p \stackrel{r}{T} G$. 
Proof. First, since $G$ operates on $F$ effectively, $\stackrel{r}{T} G$ operates on $\stackrel{r}{T} F$ effectively by virtue of Theorem 3.5. Let $\left\{U_{\alpha}\right\}$ be an open covering of $M$ such that $E$ is trivial over $U_{\alpha}$ with trivialization $\Psi_{\alpha}: \pi^{-1}\left(U_{\alpha}\right) \rightarrow U_{\alpha} \times F$ and with transition functions $g_{\alpha \beta}$, i.e. $\Psi_{\alpha} \circ \Psi_{\beta}^{-1}(x, y)=\left(x, g_{\alpha \beta}(x) \cdot y\right)$ for $x \in U_{\alpha} \cap U_{\beta}$ and $y \in F$. Clearly $\left\{\stackrel{r}{T} U_{\alpha}\right\}$ is an open covering of $\stackrel{r}{T} M$ and $\stackrel{r}{T} \Psi_{\alpha}$ is a diffeomorphism of $(\stackrel{r}{T} \pi)^{-1}\left(\stackrel{r}{T} U_{\alpha}\right)$ onto $\stackrel{r}{T} U_{\alpha} \times \stackrel{r}{T} F$. We shall verify the following

$$
\left(\stackrel{r}{T} \Psi_{\alpha}\right) \circ\left(\stackrel{r}{T} \Psi_{\beta}\right)^{-1}\left([\varphi]_{r},[\psi]_{r}\right)=\left([\varphi]_{r},\left(\left(\stackrel{r}{T} g_{\alpha \beta}\right)[\varphi]_{r}\right) \cdot[\psi]_{r}\right)
$$

for $[\varphi]_{r} \in \stackrel{r}{T}\left(U_{\alpha} \cap U_{\beta}\right)$ and $[\psi]_{r} \in \stackrel{r}{T} F$. We denote by $\rho: G \times F \rightarrow F$ the operation of $G$ on $F$ and by $\pi_{1}: U_{\alpha} \cap U_{\beta} \times F \rightarrow U_{\alpha} \cap U_{\beta}$ (resp. $\pi_{2}: U_{\alpha} \cap U_{\beta} \times F \rightarrow F$ ) the projection. Similarly we define $\tilde{\pi}_{1}: \stackrel{r}{T}\left(U_{\alpha} \cap U_{\beta}\right) \times T F \rightarrow \stackrel{r}{T}\left(U_{\alpha} \cap U_{\beta}\right)$ and $\tilde{\pi}_{2}$. Then, we have the following equalities

$$
\pi_{1} \circ \Psi_{\alpha} \circ \Psi_{\beta}^{-1}=\pi_{1}, \pi_{2} \circ \Psi_{\alpha} \circ \Psi_{\beta}^{-1}=\rho \circ\left(g_{\alpha \beta} \times 1_{F}\right) .
$$

Taking the tangent to (6.2) of order $r$, we get, by Propositions 1.6 and 1. 7, the following

$$
\left\{\begin{array}{l}
\tilde{\pi}_{1} \circ T \Psi_{\alpha} \circ T \Psi_{\beta}^{-1}=\tilde{\pi}_{1}, \\
\tilde{\pi}_{2} \circ \stackrel{r}{T} \Psi_{\alpha} \circ \stackrel{r}{T} \Psi_{\beta}^{-1}=\stackrel{r}{T} \rho \circ\left(T g_{\alpha \beta} \times 1_{T F}^{r}\right),
\end{array}\right.
$$

which proves (6. 1). Therefore, we have proved that $\stackrel{r}{T} E$ is a fibre bundle with transition functions $\left\{\stackrel{r}{T} g_{\alpha \beta}\right\}$. Q.E.D.

Definition 6. 2. We shall call the fibre bundle $\stackrel{r}{T} E(\stackrel{r}{T} M, \stackrel{r}{T} \pi, \stackrel{r}{T} F, \stackrel{r}{T} G)$ the tangential fibre bundle to $E$ of order $r$.

Let $P(M, \pi, G)$ be a principal fibre bundle with bundle space $P$, base $M$, projection $\pi$ and structure group $G$, and let $\left\{U_{\alpha}\right\}$ be an open covering of $M$ such that $P$ is trivial over $U_{\alpha}$ and let $\left\{g_{\alpha \beta}\right\}$ be the transition function with respect to this covering $\left\{U_{\alpha}\right\}$. We denote such a principal fibre bundle by $P(M, \pi, G)=\left\{U_{\alpha}, g_{\alpha \beta}\right\}$. (For the general theory of fibre bundles, see [5]). Then, by the proof of Proposition 6.1 we obtain the following

Corollary 6. 3. From a principal fibre bundle $P(M, \pi, G)=\left\{U_{\alpha}, g_{\alpha \beta}\right\}$ we get a principal fibre bundle $\stackrel{r}{T} P(\stackrel{r}{T} M, \stackrel{r}{T} \pi, \stackrel{r}{T} G)=\left\{\stackrel{r}{T} U_{\alpha}, \stackrel{r}{T} g_{\alpha \beta}\right\}$ for any positive integer $r$. 


\section{\$7. Imbedding of $\stackrel{\boldsymbol{r}}{\boldsymbol{T}} \boldsymbol{F} \boldsymbol{\text { into }} \underset{\boldsymbol{F}}{\boldsymbol{T}} \boldsymbol{\boldsymbol { r }} \boldsymbol{\text { I }}$.}

Let $F(M)(M, \pi, G L(n))$ be the frame bundle of an $n$-dimensional manifold $M$ as in [4]. We shall prove the following

THEOREM 7. 1. For any manifold $M$, there is a canonical injection $j_{M}^{(r)}: \stackrel{r}{T F M}$ $\rightarrow F \stackrel{r}{T M}$ of the tangential fibre bundle $\stackrel{r}{T} F M$ to $F M$ of order $r$ into the frame bundle of $\stackrel{r}{T M}$ such that $j_{M}^{(r)}(x \cdot g)=j_{M}^{(r)}(x) \cdot j_{n}^{(r)}(g)$ for $x \in \stackrel{r}{T} F M, g \in \stackrel{r}{T} G L(n)$ and that the following diagram is commutative:

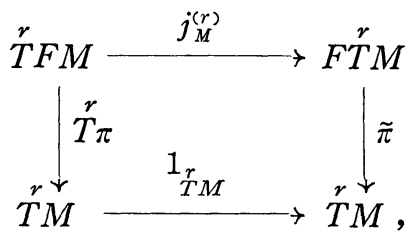

where $\pi: F M \rightarrow M($ resp. $\tilde{\pi}: \stackrel{r}{F T} M \rightarrow \stackrel{r}{T} M)$ is the projection.

Proof. We shall use the same notations as in the proof of Theorem 2. 4 [4]. We denote by $J_{\alpha \beta}^{(r)}$ the Jacobian matrix with respect to the coordinate systems $\left\{x_{\alpha, i}^{(\nu)} \mid i=1, \cdots, n ; \nu=0,1, \cdots, r\right\}$ and $\left\{x_{\beta, i}^{(\nu)} \mid i=1, \cdots, n\right.$; $\nu=0,1, \cdots, r\}$. Using the same arguments as the proof of Theorem 2.4 [4], in order to prove the Theorem 7.1, it is sufficient to verify the following relation:

$$
J_{\alpha \beta}^{(r)}=j_{n}^{(r)} \circ \stackrel{r}{T} J_{\alpha \beta} \text { on } \stackrel{r}{T}\left(U_{\alpha}\right) \cap \stackrel{r}{T}\left(U_{\beta}\right) .
$$

We shall prove (7.1) only for $r=2$, since the proof for the case $r \geqq 3$ is similar. Put $\stackrel{(\nu)}{x_{i}}=\stackrel{(\nu)}{x_{\alpha, i}}$ and $\stackrel{(\nu)}{y_{i}}=\stackrel{(\nu)}{x}_{\beta, i}$ for $i=1,2, \cdots, n ; \nu=0,1, \cdots, r$. By expressing $y_{i}$ as a function $f_{i}\left(x_{1}, \cdots, x_{n}\right)$ of $x_{1}, \cdots, x_{n}$, we get from (1. 3) the following relation:

$$
J_{\alpha \beta}^{(2)}=\left(\begin{array}{ccc}
J_{\alpha \beta} & 0 & 0 \\
\dot{J}_{\alpha \beta} & J_{\alpha \beta} & 0 \\
\ddot{J}_{\alpha \beta} & \dot{J}_{\alpha \beta} & J_{\alpha \beta}
\end{array}\right)
$$

where $\dot{J}_{\alpha \beta}=\left(\dot{J}_{k}^{i}\right)$ with $\dot{J}_{k}^{i}=\sum_{j} \frac{\partial^{2} f_{i}}{\partial x_{j} \partial x_{k}} \dot{x}_{j}$ and $\ddot{J}_{\alpha \beta}=\left(\ddot{J}_{k}^{i}\right)$ with $\ddot{J}_{k}^{i}=\frac{\dot{1}}{2} \sum_{j, l}$ $\frac{\partial^{3} f_{i}}{\partial x_{j} \partial x_{k} \partial x_{l}} \dot{x}_{j} \dot{x}_{i}+\sum_{j} \frac{\partial^{2} f_{i}}{\partial x_{j} \partial x_{k}} \ddot{x}_{j}$. Putting $J_{k}^{i}=\frac{\partial f_{i}}{\partial x_{k}}$ we get the following 


$$
J_{k}^{i}=\sum_{j} \frac{\partial J_{k}^{i}}{\partial x_{j}} \dot{x}_{j}, \quad \ddot{J}_{k}^{i}=\frac{1}{2} \sum_{j, l} \frac{\partial^{2} J_{k}^{i}}{\partial x_{j} \partial x_{l}} \dot{x}_{j} \dot{x}_{l}+\sum_{j} \frac{\partial J_{k}^{i}}{\partial x_{j}} \ddot{x}_{j}
$$

Now, consider the map $J=J_{\alpha \beta}: U_{\alpha} \cap U_{\beta} \rightarrow G L(n)$. We can calculate the coordinates $\left(y_{j}^{i} \mid i, j=1, \cdots, n ; \nu=0,1,2\right)$ of the image of $\left(x_{i} \mid i=1, \cdots, n\right.$; $\nu=0,1,2)$ by the map $\stackrel{2}{T} J$ as follows:

$$
\left\{\begin{array}{l}
\stackrel{(0)}{y_{j}^{i}}=J_{j}^{i}(x), \stackrel{(1)}{y_{j}^{i}}=\sum_{k} \frac{\partial J_{j}^{i}}{\partial x_{k}} \dot{x}_{k}, \\
\stackrel{(2)}{y_{j}^{i}}=\frac{1}{2} \sum_{k, l} \frac{\partial^{2} J_{j}^{i}}{\partial x_{k} \partial x_{l}} \dot{x}_{k} \dot{x}_{l}+\sum_{k} \frac{\partial J_{j}^{i}}{\partial x_{k}} \ddot{x}_{k}
\end{array}\right.
$$

By Proposition 5. 3 and (7. 4), (7. 3) we obtain

$$
j_{n}^{(2)} \circ \stackrel{2}{T} J_{\alpha \beta}=J_{\alpha \beta}^{(2)} \text { on } \stackrel{2}{T}\left(U_{\alpha}\right) \cap \stackrel{2}{T}\left(U_{\beta}\right)
$$

\section{§8. Prolongations of $G$-structures to tangent bundles of order $\boldsymbol{r}$.}

Definition 8. 1. Let $G$ be a Lie subgroup of $G L(n)$. We denote by $G^{(r)}$ the image of $\stackrel{r}{T} G$ by the homomorphism $j_{n}^{(r)}$, j.e.

$$
\left.G^{(r)}=j_{n}^{(r)} \stackrel{r}{T} G\right)
$$

Clearly, $G^{(r)}$ is a Lie subgroup of $G L(n(r+1))$.

Let $P(M, \pi, G)$ be a $G$-structuure on $M$ (for the general theory of $G$ structures see, for instance [1], [2], [4] or [6]). We denote by $\pi^{(r)}$ the restriction of the projection $\pi: F \stackrel{r}{T} M \rightarrow \stackrel{r}{T} M$ to the subbundle $\left.P^{(r)}=j_{M}^{(r)} \stackrel{r}{T} P\right)$. Then we obtain a $G^{(r)}$-structure $P^{(r)}\left(\stackrel{r}{T} M, \pi^{(r)}, G^{(r)}\right)$ on the tangent bundle $\stackrel{r}{T} M$ to $M$ of order $r$. We shall call $P^{(r)}$ the prolongation of order $r$ of the $G$-structure $P$ to the tangent bundle $\stackrel{r}{T} M$ to $M$ of order $r$.

We can easily see the following

Proposition 8.2. If $M$ is completely parallelizable, then $\stackrel{r}{T} M$ is also completely parallelizable.

Proposition 8. 3. There is a canonical bundle homomorphism $\beta_{s}^{r}$ of $P^{(r)}$ into $P^{(s)}$ for $r>s$, i.e. the following diagram 


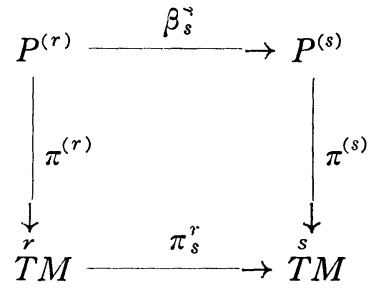

is commutative and there is a canonical homomorphism $h_{s}^{r}: G^{(r)} \rightarrow G^{(s)}$ such that

$$
\beta_{s}^{r}(x \cdot a)=\beta_{s}^{r}(x) \cdot h_{s}^{r}(a)
$$

for $x \in P^{(r)}$ and $a \in G^{(r)}$.

\section{§. Prolongations of isomorphisms of $G$-structures.}

Theorem 9. 1. Let $M$ and $M^{\prime}$ be two manifolds and $f: M \rightarrow M^{\prime}$ be a diffeomorphism betw en them. Then, we have the following commutative diagram:

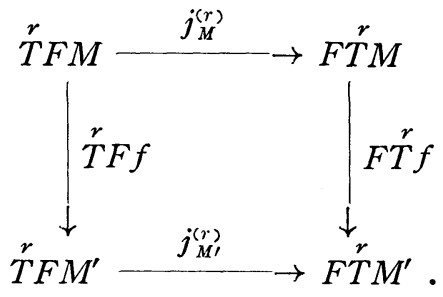

Proof. We use the same notations $\Phi_{\alpha}, \Phi_{\alpha}^{\prime}, f_{\alpha}$ as in the proof of Theorem 4. 2 [4]. On the other hand, let

$$
\begin{aligned}
& \Psi_{\alpha}: \stackrel{r}{T} U_{\alpha} \times G L(n(r+1)) \rightarrow F \stackrel{r}{T} U_{\alpha} \\
& \Psi_{\alpha}^{\prime}: \stackrel{r}{T} V_{\alpha} \times G L(n(r+1)) \rightarrow F \stackrel{r}{T} V_{\alpha}
\end{aligned}
$$

be the local trivializations of $\underset{F T}{T} M$ (resp. $\left.F \stackrel{r}{F} M^{\prime}\right)$ over $\stackrel{r}{T} U_{\alpha}\left(\operatorname{resp} . \stackrel{r}{T} V_{\alpha}\right)$ induced by the coordinate system on $U_{\alpha}$ (resp. $\left.V_{\alpha}\right)$. Define $f_{\alpha}^{(r)}: \stackrel{r}{T} U_{\alpha} \times$ $G L(n(r+1)) \rightarrow \stackrel{r}{T} V_{\alpha} \times G L(n(r+))$ by the following

$$
f_{\alpha}^{(r)}=\Psi_{\alpha}^{\prime-1} \circ F T^{r} f \circ \Psi_{\alpha} .
$$

Let $j_{\alpha}^{(r)}=1_{r U} \times j_{n}^{(r)}$ and $j_{\alpha}^{\prime(r)}=1_{r V_{\alpha}} \times j_{n}^{(r)}$. By the same arguments - as the proof of Th. 4. 2 [4], in order to prove the Theorem 9.1, it is now suffficient to prove the commutativity of the following diagram: 
(9. 1)

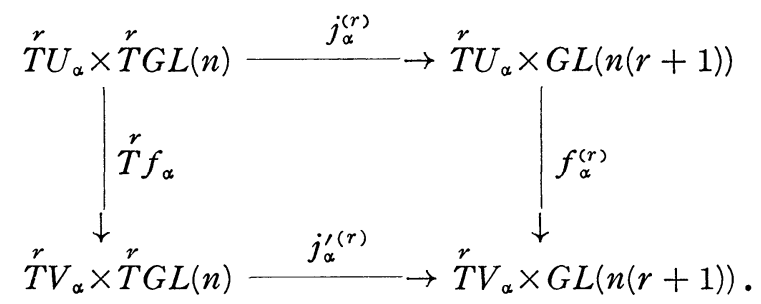

We shall prove the commutativity of (9.1) only for the case $r=2$, since the case for $r \geqq 3$ is similar. Using the same notations $y_{i}, f_{i}(x), w_{i}^{k}, z_{i}^{k}$ as in Th. 4. 2 [4] (we use $y_{i}$ instead of $y^{i}$, etc), we introduce the notations $f_{\kappa}(x), x_{\kappa}, y_{\kappa}$ for $\kappa=1,2, \cdots, 3 n$ by the following

$$
\left\{\begin{array}{l}
f_{i+n}=\sum \frac{\partial f_{i}}{\partial x_{k}} \dot{x}_{k}, \\
f_{i+2 n}=\frac{1}{2} \sum \frac{\partial^{2} f_{i}}{\partial x_{k} \partial x_{l}} \dot{x}_{k} \dot{x}_{l}+\sum \frac{\partial f_{i}}{\partial x_{k}} \ddot{x}_{k}, \\
x_{i+n}=\dot{x}_{i}, \quad x_{i+2 n}=\ddot{x}_{i}, \quad y_{i+n}=\dot{y}_{i}, \quad y_{j+2 n}=\ddot{y}_{i}
\end{array}\right.
$$

for $i=1,2, \cdots, n$. Let $\left\{x_{\kappa}, \tilde{w}_{\lambda}^{\kappa} \mid \kappa, \lambda=1,2, \cdots, 3 n\right\} \quad$ (resp. $\left\{y_{\kappa}, \tilde{z}_{\lambda}^{\kappa} \mid \kappa, \lambda=1,2\right.$, $\cdots, 3 n\})$ be the coordinate system on $F \stackrel{2}{T U_{\alpha}}$ (resp. $F \stackrel{2}{T} V_{\alpha}$ ) induced by the coordinate system $\left\{x_{\kappa}\right\}$ (resp. $\left\{y_{\kappa}\right\}$ ). Now since the map $f_{\alpha}: U_{\alpha} \times G L(n) \rightarrow$ $V_{\alpha} \times G L(n)$ is expressed as follows:

$$
f_{\alpha}: y_{i}=f_{i}(x), z_{i}^{j}=\sum w_{i}^{k} \frac{\partial f_{j}}{\partial x_{k}}(i, j=1,2, \cdots, n),
$$

we obtain the expression of $\stackrel{2}{T} f_{\alpha}$ as follows:

(9. 4)

$$
\left\{\begin{array}{l}
y_{i}=f_{i}(x), z_{i}^{j}=\sum_{k} w_{i}^{k} \frac{\partial f_{j}}{\partial x_{k}}, \\
\dot{y}_{i}=\sum_{k} \frac{\partial f_{i}}{\partial x_{k}} \dot{x}_{k}, \\
\dot{z}_{i}^{j}=\sum_{k, l} w_{i}^{k} \frac{\partial^{2} f_{j}}{\partial x_{k} \partial x_{l}} \dot{x}_{l}+\sum_{k} \frac{\partial f_{i}}{\partial x_{k}} \dot{w}_{i}^{k}, \\
\ddot{y}_{i}=\frac{1}{2} \sum_{k, l} \frac{\partial^{2} f_{i}}{\partial x_{k} \partial x_{l}} \dot{x}_{k} \dot{x}_{l}+\sum_{k} \frac{\partial f_{i}}{\partial x_{k}} \ddot{x}_{k}, \\
\ddot{z}_{i}^{j}=\frac{1}{2}\left(\sum_{k, l, m} w_{i}^{k} \frac{\partial^{3} f_{j}}{\partial x_{k} \partial x_{l} \partial x_{m}} \dot{x}_{l} \dot{x}_{m}+\sum_{k, l} \frac{\partial^{2} f_{j}}{\partial x_{k} \partial x_{l}} \dot{x}_{l} \dot{w}_{i}^{k}\right) \\
+\sum_{k, l} w_{i}^{k} \frac{\partial^{2} f_{j}}{\partial x_{k} \partial x_{l}} \ddot{x}_{l}+\frac{1}{2} \sum_{k, l} \frac{\partial^{2} f_{j}}{\partial x_{k} \partial x_{l}} \dot{x}_{l} \dot{w}_{i}^{k}+\sum_{k} \frac{\partial f_{j}}{\partial x_{k}} \ddot{w}_{i}^{k}
\end{array}\right.
$$


By Proposition 5. 3 we get the following

$$
\left(j_{\alpha}^{\prime(2)} \circ \stackrel{2}{T} f_{\alpha}\right)\left(x_{\kappa}, w_{\lambda}^{\kappa}\right)=\left(y_{\kappa},\left(\begin{array}{ccc}
z_{i}^{j} & 0 & 0 \\
\dot{z}_{i}^{j} & z_{i}^{j} & 0 \\
\ddot{z}_{i}^{j} & \dot{z}_{i}^{J} & z_{i}^{j}
\end{array}\right)\right),
$$

where $y_{\kappa}$ and $z_{\lambda}^{\kappa}$ are given by (9.4).

On the other hand, since $f: U_{\alpha} \rightarrow V_{\alpha}$ is expressed by $y_{i}=f_{i}\left(x_{1}, \cdots, x_{n}\right)$ $(i=1, \cdots, n)$, we have the expression of $\stackrel{2}{T} f$ as follows:

$$
\stackrel{2}{T f}:\left\{\begin{array}{l}
y_{i}=f_{i}(x), \quad \dot{y}_{i}=\sum_{k} \frac{\partial f_{i}}{\partial x_{k}} \dot{x}_{k}, \\
\ddot{y}_{i}=\frac{1}{2} \sum_{k, l} \frac{\partial^{2} f_{i}}{\partial x_{k} \partial x_{l}} \dot{x}_{k} \dot{x}_{l}+\sum_{k} \frac{\partial f_{i}}{\partial x_{j}} \ddot{x}_{k} .
\end{array}\right.
$$

Therefore, we get the expression of $f_{\alpha}^{(2)}$ as follows:

$$
f_{\alpha}^{(2)}:\left\{\begin{array}{l}
y_{i}=f_{i}(x), \quad \dot{y}_{i}=\sum_{k} \frac{\partial f_{i}}{\partial x_{k}} \dot{x}_{k}, \\
\ddot{y}_{i}=\frac{1}{2} \sum_{k, l} \frac{\partial^{2} f_{i}}{\partial x_{k} \partial x_{l}} \dot{x}_{k} \dot{x}_{l}+\sum_{k} \frac{\partial f_{i}}{\partial x_{k}} \ddot{x}_{k}, \\
\tilde{z}_{\lambda}^{\kappa}=\sum_{\mu=1}^{3 n} \tilde{w}_{\lambda}^{\mu} \frac{\partial f_{k}}{\partial x_{\mu}}
\end{array}\right.
$$

for $\kappa, \lambda=1,2, \cdots, 3 n$ and $i=1,2, \cdots, n$. Now, we calculate $\tilde{z}_{\lambda}^{\kappa}$ by (9.2) as follows:

$$
\begin{aligned}
\tilde{z}_{\kappa}^{j}= & \sum_{k} \tilde{w}_{\kappa}^{k} \frac{\partial f_{j}}{\partial x_{k}}, \\
\tilde{z}_{\kappa}^{n+j}= & \sum_{k, l} \tilde{w}_{\kappa}^{k} \frac{\partial^{2} f_{j}}{\partial x_{l} \partial x_{k}} \dot{x}_{l}+\sum_{k} \tilde{w}_{\kappa}^{n+k} \frac{\partial f_{j}}{\partial x_{k}}, \\
\tilde{z}_{\kappa}^{2 n+j} & =\sum_{k} \tilde{w}_{\kappa}^{k} \frac{\partial f_{2 n+j}}{\partial x_{k}} \sum_{k} \tilde{w}_{\kappa}^{n+k} \frac{\partial f_{2 n+j}}{\partial \dot{x}_{k}} \sum_{k} \tilde{w}_{\kappa}^{2 n+k} \frac{\partial f_{2 n+j}}{\partial \ddot{x}_{k}} \\
& =\sum_{k} \tilde{w}_{\kappa}^{k}\left(\frac{1}{2} \sum_{l, m} \frac{\partial^{3} f_{j}}{\partial x_{m} \partial x_{l} \partial x_{k}} \dot{x}_{m} \dot{x}_{l}+\sum_{l} \frac{\partial^{2} f_{j}}{\partial x_{l} \partial x_{k}} \ddot{x}_{l}\right) \\
& +\sum_{k, l} \tilde{w}_{\kappa}^{n+k} \frac{\partial^{2} f_{j}}{\partial x_{k} \partial x_{l}} \dot{x}_{k}+\sum_{k} \tilde{w}_{\kappa}^{2 n+k} \frac{\partial f_{j}}{\partial x_{k}} .
\end{aligned}
$$

for $\kappa=1,2, \cdots, 3 n$ and $j=1,2, \cdots, n$. By Proposition 5.3 and the above calculations, we have the following equalities: 


$$
\begin{aligned}
& f_{\alpha}^{(2)} \circ j_{\alpha}^{(2)}\left(x_{\mu} ; w_{i}^{k}, \dot{w}_{i}^{k}, \ddot{w}_{i}^{k}\right)=f_{\alpha}^{(2)}\left(x_{\mu} ;\left(\begin{array}{ccc}
w_{i}^{k} & 0 & 0 \\
\dot{w}_{i}^{k} & w_{i}^{k} & 0 \\
\ddot{w}_{i}^{k} & \dot{w}_{i}^{k} & w_{i}^{k}
\end{array}\right)\right) \\
& =\left(f_{i}(x), \dot{y}_{i}, \ddot{y}_{i} ;\left(\begin{array}{ccc}
\tilde{z}_{i}^{j} & 0 & 0 \\
\tilde{z}_{i}^{n+j} & \tilde{z}_{i}^{j} & 0 \\
\tilde{z}_{i}^{n+j} & \tilde{z}_{i}^{n+j} & \tilde{z}_{i}^{j}
\end{array}\right)\right) \text {, }
\end{aligned}
$$

where we see that $\tilde{z}_{i}^{j}=\sum_{k} w_{i}^{k} \frac{\partial f_{j}}{\partial x_{k}}, \quad \tilde{z}_{i}^{n+j}=\sum_{k, l} w_{i}^{k} \frac{\partial^{2} f_{j}}{\partial x_{l} \partial x_{k}} \dot{x}_{l}+\sum_{k} \dot{w}_{i}^{k} \frac{\partial f_{i}}{\partial x_{k}} \quad$ and $\tilde{z}_{j}^{2 n+j}=\sum_{k} w_{i}^{k}\left(\frac{1}{2} \sum_{l, m} \frac{\partial^{3} f_{j}}{\partial x_{m} \partial x_{l} \partial x_{k}} \dot{x}_{m} \dot{x}_{l}+\sum_{l} \frac{\partial^{2} f_{j}}{\partial x_{l} \partial x_{k}} \ddot{x}_{l}\right)+\sum_{k, l} \dot{w}_{i}^{k} \frac{\partial^{2} f_{j}}{\partial x_{k} \partial x_{l}} \dot{x}_{l}+\sum_{k}$ $\ddot{w}_{i}^{k} \frac{\partial f_{j}}{\partial x_{k}}=\ddot{z}_{i}^{j}$. Therefore, we obtain, by (9.5) and (9.6), the commutativity of (9. 1) for $r=2$.

By the same arguments as the proof of Th. 4. 3 [4] we can prove the following

Theorem 9.2. Let $\Phi$ be a diffeomorphism of a manifold $M$ onto a manifold $M^{\prime}$. Let $P\left(\right.$ resp. $\left.P^{\prime}\right)$ be a $G$-structure on $M$ (resp. $\left.M^{\prime}\right)$. Then $\Phi$ is an isomorphism of $P$ with $P^{\prime}$ if an only if $\stackrel{r}{T \Phi}$ is an isomorphism of $P^{(r)}$ with $P^{\prime(r)}$.

Corollary 9. 3. Let $\Phi$ be a diffeomorphism of $M$ onto itself, and let $P$ be a G-structure on $M$. Then $\Phi$ is an automorphism of $P$ if and only if $\stackrel{r}{T} \Phi$ is an automorphism of the prolongation $P^{(r)}$ of order $r$.

\section{\$10. Integrability of prolongations of $G$-structures.}

In this section, we shall prove that the prolongation of an integrable $G$-structure (see Def. 5. 1 [4]) of order $r$ is also integrable and vice versa.

Proposition 10.1. Let $\left\{x_{1}, \cdots, x_{n}\right\}$ be a local coordinate system on a neighborhood $U$ in $M$, on which we give a G-structure $P$. Let $\phi$ be a cross section of $P$ over $U$, which is expressed by $\phi(x)=\left(\cdots, \Sigma \phi_{j}^{i}(x)\left(\partial / \partial x_{i}\right)_{x}, \cdots\right)$ for $x \in U$. Define $\phi^{(r)}$ by $\phi^{(r)}=j_{M}^{(r)} \circ \stackrel{r}{T} \phi$. Then $\phi^{(r)}$ is a cross section of the prolongation $P^{(r)}$ over $\stackrel{r}{T} U$ and is expressed with respect to the induced coordinate system $\left\{x_{i} \mid i=1\right.$, $\cdots, n ; \nu=0,1, \cdots, r\}$ as follows:

$$
\phi^{(r)}\left(\cdots, x_{i}^{(\nu)}, \cdots\right)=\left(\cdots, \sum_{i=1}^{n} \phi_{j}^{i}(x)\left(\frac{\partial}{\partial x_{i}}\right)_{X}+\sum_{\mu>\nu} \sum_{i=1}^{n} F_{j, \mu}^{i, \nu}(X)\left(\frac{\partial}{\partial x_{i}}\right)_{X}, \cdots\right),
$$


where $X=\left(\cdots, x_{i}, \cdots\right) \in \stackrel{r}{T} U$ and $F_{j, \mu}^{i, \nu}(X)$ is a polynomial of $\stackrel{(\lambda)}{x_{k}}(\lambda \geqq \mu ; k=1$, $\cdots, n)$ without constant term and with coefficients, which are partial derivatives of $\phi_{m}^{l}(l, m=1, \cdots, n)$.

Proof. Let $\pi: F(M) \rightarrow M$ and $\tilde{\pi}: \stackrel{r}{F T} M \rightarrow \stackrel{r}{T} M$ be the projections. Let $\Phi_{U}$ and $\Psi_{U}$ be the local trivialization of $F M$ and $F \stackrel{r}{T} M$ over $U$ and $\stackrel{r}{T} U$, respectively. We see that

$$
j_{M}^{(r)} \mid \stackrel{r}{T} F M=\Psi_{U} \circ\left(1_{r U} \times j_{n}^{(r)}\right) \circ\left(\stackrel{r}{T} \Phi_{U}\right)^{-1} .
$$

Using Proposition 1. 6, we have the following equalities:

$$
\tilde{\pi} \circ \phi^{(r)}=\tilde{\pi} \circ j_{M}^{(r)} \stackrel{r}{T} \phi=\stackrel{r}{T} \pi \circ \stackrel{r}{T} \phi=\stackrel{r}{T}(\pi \circ \phi)=\stackrel{r}{T} 1_{U}=1_{r}
$$

Since $\left.\phi^{(r)} \stackrel{r}{T} U\right)=j_{M}^{(r)} \circ \stackrel{r}{T} \phi(T U)=j_{M}^{(r)} \stackrel{r}{T}(\phi(U)) \subset j_{M}^{(r)} \stackrel{r}{T} P=P^{(r)}$, we see that $\phi^{(r)}$ is a cross section of $P^{(r)}$ over $\stackrel{r}{T} U$.

We shall prove (10.1) only for the case $r=2$, since the case $r \geqq 3$ is similar. Put $f(x)=\left(\phi_{j}^{i}(x)\right) \in G L(n)$ for $x \in U$, then we have $\Phi_{U}^{-1} \circ \phi=\left(1_{U}, f\right)$. Hence, we have $\left.\phi^{(2)}=\Psi_{U} \circ\left(\underline{1}_{T U} \times j_{n}^{(2)}\right) \circ \stackrel{2}{T} \Phi\right)^{-1} \circ \stackrel{2}{T} \phi=\Psi_{U} \circ\left({\underset{T}{T} U}_{T} \times j_{n}^{(2)}\right) \circ \stackrel{2}{T}\left(1_{U}, f\right)=$ $\Psi_{U} \circ\left(1_{2 U} \times j_{n}^{(2)} \circ \stackrel{2}{T} f\right)$. Therefore, using the expression (1.2) of $T^{2} f$ and Proposition 5.3 we get the expression of $\phi^{(2)}$ as follows:

$$
\begin{gathered}
\phi^{(2)}(x, \dot{x}, \ddot{x})=\Psi_{U}\left((x, \dot{x}, \ddot{x}) ;\left(\begin{array}{ccc}
\phi_{j}^{i} & 0 & 0 \\
\dot{\phi} & \phi_{j}^{i} & 0 \\
\ddot{\phi}_{j}^{i} & \dot{\phi}_{j}^{i} & \phi_{f}^{i}
\end{array}\right)\right) \\
=\left(\cdots, \sum_{i}\left(\phi_{j}^{i}\left(\frac{\partial}{\partial x_{i}}\right)_{X}+\dot{\phi}_{j}^{i}\left(\frac{\partial}{\partial \dot{x}_{i}}\right)_{X}+\ddot{\phi}_{j}^{i}\left(\frac{\partial}{\partial \ddot{x}_{i}}\right)_{X}\right), \cdots,\right. \\
\left.\sum_{i}\left(\phi_{j}^{i}\left(\frac{\partial}{\partial \dot{x}_{i}}\right)_{X}+\dot{\phi}_{j}^{i}\left(\frac{\partial}{\partial \ddot{x}_{i}}\right)_{X}\right), \cdots, \sum_{i} \phi_{j}^{i}\left(\frac{\partial}{\partial \ddot{x}_{i}}\right)_{X}, \cdots\right),
\end{gathered}
$$

where

$$
\dot{\phi}_{j}^{i}=\sum_{k} \frac{\partial \dot{\phi}_{j}^{i}}{\partial x_{k}} \dot{x}_{k}, \ddot{\phi}_{j}^{i}=\frac{1}{2} \sum_{k, l} \frac{\partial^{2} \phi_{j}^{i}}{\partial x_{k} \partial x_{l}} \dot{x}_{k} \dot{x}_{l}+\sum_{k} \frac{\partial \phi_{j}^{i}}{\partial x_{k}} \ddot{x}_{k} .
$$

These functions $\dot{\phi}_{j}^{i}$ and $\ddot{\phi}_{j}^{i}$ have the properites stated in the proposition. Thus the proposition is proved.

Q.E.D.

Remark 10.2. By the properties of the functions $F_{j, \mu}^{i, \nu}(X)$, we see that $F_{j, \mu}^{i, \nu}$ vanishes if the functions $\phi_{m}^{l}$ are constants for $l, m=1,2, \cdots, n$. The 
function $F_{j, \mu}^{i, \nu}(X)$ also vanishes at $X=\left(\cdots, x_{i}^{(\nu)}, \cdots\right)$ with $\stackrel{(\lambda)}{x_{k}}=0$ for all $\lambda \geqq \mu$ and $k=1, \cdots, n$, since $F_{j, \mu}^{i, \nu}$ is a polynomial of $x_{k}^{(\lambda)}$ without constant term.

Theorem 10.3. Let $P$ be a G-structure on a manifold $M$. Then, $P$ is integrable if and only if the prolongation $P^{(r)}$ of $P$ order $r$ is integrable for any $r$.

Proof. Suppose $P$ is integrable. Let $x_{0} \in M$ be any point of $M$ and let $\left\{x_{1}, \cdots, x_{n}\right\}$ be a local coordinate system on a neighborhood $U$ of $x_{0}$ such that

$$
\phi(x)=\left(\cdots,\left(\frac{\partial}{\partial x_{i}}\right)_{x}, \cdots\right) \in P \text { for any } x \in U .
$$

Then, by Proposition 10.1 and Remark 10.2, $\phi^{(r)}$ is a cross section of $P^{(r)}$ and is expressed with respect to the induced coordinate system $\left\{\stackrel{(\nu)}{x_{i}} \mid i=1\right.$, $\cdots, n ; \nu=0,1, \cdots, r\}$ as follows: $\phi^{(r)}\left(\cdots, x_{i}^{(\nu)}, \cdots\right)=\left(\cdots,\left(\partial / \partial x_{i}\right)_{X}, \cdots\right)$ for $X=\left(\cdots, x_{i}, \cdots\right) \in \stackrel{r}{T} U$. Since $\phi^{(r)}(X) \in P^{(r)}$ and since $x_{0}$ is arbitrary, we have proved that $P^{(r)}$ is integrable.

Conversely, suppose $P^{(r)}$ is integrable for some $r$. To prove that $P$ is integrable, we use the same arguments as the proof of Prop. 5. 5 [4]. Take a point $p \in M$ and take a coordinate neighborhood $U$ of $p$ with coordinate system $\left\{x_{1}, \cdots, x_{n}\right\}$ such that there is a local cross section $\phi: U \rightarrow P$ of $P$ over $U$. Then, by Proposition $10.1, \phi^{(r)}=j_{M}^{(r)} \circ \stackrel{r}{T} \phi$ is a cross section of $P^{(r)}$ over $\stackrel{r}{T} U$. Now, let $X_{0}$ be the element of $\stackrel{r}{T} U$ having coordinates $\left\{\stackrel{(\nu)}{x_{i}}\right\}$ with $x_{i}=x_{i}(p)$ and $\stackrel{(\nu)}{x_{i}}=0$ for all $\nu \geqq 1$ and $i=1, \cdots, n$. Since $P^{(r)}$ is integrable, there can be found a coordinate neighborhood $\tilde{U}$ of $X_{0}$ with coordinate system $\left\{y_{1}, y_{2}, \cdots, y_{N}\right\}(N=n(r+1))$ such that $\tilde{U} \subset \stackrel{r}{T} U$ and that, if we define $\tilde{\phi}_{0}$ by $\tilde{\phi}_{0}(X)=\left(\left(\partial / \partial y_{1}\right)_{X}, \cdots,\left(\partial / \partial y_{N}\right)_{X}\right), \quad \tilde{\phi}_{0}$ is a cross section of $P^{(r)}$ over $\tilde{U}$. Since $\phi^{(r)} \mid \tilde{U}$ and $\tilde{\phi}_{0}$ are both cross sections of $P^{(r)}$ over $\tilde{U}$, there exists a map $\tilde{g}: \tilde{U} \rightarrow G^{(r)}$ such that

$$
\phi^{(r)}(X)=\tilde{\phi}_{0}(X) \cdot \tilde{g}(X)
$$

holds for $X \in \tilde{U}$. By Proposition 5. 3, there is a map $g: \tilde{U} \rightarrow G$ such that $\tilde{g}(X)$ has the following form:

$$
\tilde{g}(X)=\left(\begin{array}{ccc}
g(X) & & 0 \\
& g(X) & \\
* & & \cdot g(X)
\end{array}\right) .
$$


Since $\left\{y_{1}, \cdots, y_{N}\right\}$ and $\left\{\mathcal{\nu}_{i}^{(\nu)}\right\}$ are both coordinate systems on $\tilde{U}$ we have differentiable functions $f_{k}$ such that $y_{\kappa}=f_{k}\left(\cdots, x_{i}^{(\nu)}, \cdots\right)$ for $\left(\cdots, x_{i}, \cdots\right) \in \tilde{U}$ and $\kappa=1,2, \cdots, N$. Now if $\phi(x)=\left(\cdots, \sum_{i} \phi_{j}^{i}(x)\left(\partial / \hat{o} x_{i}\right)_{x}, \cdots\right)$ for $x \in U$, then by Proposition 10.1, (10.2) can be written as follows:

$$
\begin{aligned}
& \sum_{i} \phi_{j}^{i}(x)\left(\frac{\partial}{\partial x_{i}}\right)_{X}+\sum_{\mu, i} F_{j \mu}^{i, o}(X)\left(\frac{\partial}{\partial x_{i}}\right)_{X} \\
= & \sum_{i} g_{j}^{i}(X)\left(\frac{\partial}{\delta y_{i}}\right)_{X}+\sum_{\kappa=n+1}^{N} \tilde{g}_{j}^{\kappa}(X)\left(\frac{\partial}{\partial y_{\kappa}}\right)_{X}
\end{aligned}
$$

for $j=1,2, \cdots, n$, where $\tilde{g}(X)=\left(g_{\lambda}^{\kappa}(X)\right)$ for $X \in \tilde{U}$. Since $\left(\partial / \partial x_{i}^{(\nu)}\right)_{X}=\Sigma\left(\partial f_{\kappa}\right)$ $\left(\begin{array}{l}\nu \\ \partial x_{i}\end{array}\right) \cdot\left(\partial / \partial y_{\kappa}\right)_{X},(10.4)$ can be written as follows:

$$
\begin{aligned}
& \sum_{i, \kappa} \phi_{j}^{i} \cdot \frac{\partial f_{\kappa}}{(0)} \cdot\left(\frac{\partial}{\partial x_{i}}\right)_{X}+\sum_{i, \mu, \kappa} F_{j, \mu}^{i, o}(X) \frac{\partial f_{\kappa}}{(\mu)}\left(\frac{\partial}{\partial x_{i}}\right)_{X} \\
= & \sum_{i} g_{j}^{i}(X)\left(\frac{\partial}{\partial y_{i}}\right)_{X}+\sum_{\kappa=n+1}^{N} \tilde{g}_{j}^{\kappa}(X)\left(\frac{\partial}{\partial y_{\kappa}}\right)_{X} .
\end{aligned}
$$

Comparing the coefficients of $\left(\partial / \partial y_{k}\right)_{X}$ for $k \leqq n$ in $(10.5)$, we have

$$
\sum_{i} \phi_{j}^{i}(x) \frac{\partial f_{k}}{\partial x_{i}}+\sum_{i, \mu} F_{i, \mu}^{i, o}(X) \frac{\partial f_{k}}{\partial x_{i}}=g_{j}^{k}(X)
$$

for $j, k=1,2, \cdots, n$. Now, define maps $\bar{f}_{k}: U^{\prime} \rightarrow R$ and $\bar{g}: U^{\prime} \rightarrow G$ by $\bar{f}_{k}(x)=f_{k}(x, 0, \cdots, 0)$ and $\left(\bar{g}(x)^{-1}\right)_{j}^{i}=g_{j}^{i}(x, 0, \cdots, 0)$ for $i, j, k=1, \cdots, n$ and $x \in U^{\prime}=\pi(\tilde{U})$.

Putting $\stackrel{(\nu)}{x_{k}}=0(k=1,2, \cdots, n ; \nu=1,2, \cdots, r)$ in $(10.6)$ and using Remark 10. 2 we obtain

$$
\sum_{i} \phi_{j}^{i}(x) \frac{\partial \bar{f}_{k}}{\partial x_{i}}=\left(\bar{g}(x)^{-1}\right)_{j}^{k}
$$

Now, by the same arguments as in the proof of Prop. 5. 5 [4, pp. 88-89], we see that there exists a coordinate neighborhood $U_{0}$ of $p$ with coordinate system $\left\{\bar{x}_{1}, \cdots, \bar{x}_{n}\right\}$ such that the map $\bar{\phi}$, defined by $\bar{\phi}(x)=\left(\left(\partial / \partial \bar{x}_{1}\right)_{x} ; \cdots\right.$, $\left.\left(\partial / \partial \bar{x}_{n}\right)_{x}\right)$ for $x \in U_{0}$, is a cross section of $P$ over $U_{0}$. Thus $P$ is integrable.

Q.E.D. 


\section{\$11. Prolongations of classical $G$-structures.}

(I) $G=G L(n, C)$.

Let $J$ be a linear automorphism of $R^{2 n}$ such that $J^{2}=-1_{R^{2 n}}$ and let $G L(n, C ; J)$ be the group of all $a \in G L(2 n)$ such that $a \circ J=J \circ a$. It is easy to see that $\stackrel{r}{T} J$ is a linear automorphism of $R^{2 n(r+1)}=\stackrel{r}{T}\left(R^{2 n}\right)$ such that $\left.\stackrel{r}{T} J\right)^{2}$ $=-1$. We shall prove the following

Proposition 11. 1. If $G=G L(n, C ; J)$, then $G^{(r)} \subset G L(n(r+1), C ; \stackrel{r}{T} J)$.

Proof. Take an element $\tilde{a} \in G^{(r)}$. We have to prove that $(\tilde{a} \circ \stackrel{r}{T} J)(X)$ $=((\stackrel{r}{T} J) \circ \tilde{a})(X)$ for every $X \in \stackrel{r}{T}\left(R^{2 n}\right)$. Now, we can find maps $\varphi \in S(G)$ and $\psi \in S\left(R^{2 n}\right)$ (cf. Notations in $\$ 1$ ) such that $\tilde{a}=[\varphi]_{r}$ and $X=[\psi]_{r}$. First, it is readily seen that $\varphi \cdot(J \circ \psi)=J \circ(\varphi \cdot \psi)$ (cf. Notations in Th. 5. 1). Therefore, we have $\tilde{a}(\stackrel{r}{T} J(X))=[\varphi]_{r}([J \circ \psi)]_{r}=[\varphi \cdot[J \circ \psi)]_{r}=[J \circ(\varphi \cdot \psi)]_{r}=\stackrel{r}{T} J\left([\varphi \cdot \psi]_{r}\right)$ $=\stackrel{r}{T} J\left([\varphi]_{r} \cdot[\psi]_{r}\right)=\stackrel{r}{T} J(\tilde{a}(X))$.

By the same arguments as the proof of Theorem 6.3 [4], we obtain the following

THeOREM 11.2. (1) If a manifold $M$ has an almost complex structure, $\stackrel{r}{T M}$ has a canonical almost complex structure for every $r$.

(2) If a manifold $M$ has a complex structure, then $\stackrel{r}{T} M$ has a canonical complex structure for every $r$.

(II) $\quad G=S_{p}(m)$.

Consider a skew-symmetric non-degenerate bilinear form $f$ on $R^{2 m}$. Let $S_{p}(m, f)$ be the group of all $a \in G L(2 m)$ which leaves $f$ invariant. We denote by $\pi_{r}$ the projection of $\stackrel{r}{T} R=R^{r+1}$ onto $R$ defined by $\pi_{r}\left([\varphi]_{r}\right)=(1 / r !)$ $\left[d^{r} \varphi / d t^{r}\right]_{0}$ for $\varphi \in S(R)=C^{\infty}(R)$.

Lemma 11. 3. If $f$ is a skewe-symmetric non-degenerate bilinear form on $R^{2 m}$, then $\left.f^{(r)}=\pi_{r} \circ \stackrel{r}{T} f\right)$ is also a skew-symmetric non-degenerate bilinear form on $R^{2 m(r+1)}=\stackrel{r}{T} R^{2 m}$.

Proof. We take the skew-symmetric matrix $\left(a_{j}^{i}\right) \in G L(2 m)$ such that $f(x, y)=\sum a_{j}^{i} x_{i} y_{j}$ for $x=\left(x_{1}, \cdots, x_{n}\right)$ and $y=\left(y_{1}, \cdots, y_{n}\right)$ with $n=2 m$. Let $\left\{x_{i}\right\}$ be the induced coordinate system on $R^{n(r+1)}$. Take an element 
$[\varphi]_{r}\left(\operatorname{resp} .[\psi]_{r}\right)$ of $\stackrel{r}{T} R^{n}$ with coordinates $\left\{\stackrel{(\nu)}{x_{i}}\right\}$ (resp. $\left.\left\{\stackrel{(\nu)}{y_{i}}\right\}\right)$. We can assume that $\varphi(t)=\left(\cdots, \sum_{\nu=0}^{r(\nu)} x_{i} t^{\nu}, \cdots\right)$ and $\psi(t)=\left(\cdots, \sum_{\nu=0}^{r(\nu)} y t^{\nu}, \cdots\right)$. It is now straightforward to see that the following equality holds:

$$
f^{(r)}\left([\varphi]_{r},\left[\psi_{r}\right]\right)=\sum_{i, j} \sum_{\nu=0}^{r} a_{i j}^{(\nu)(r-\nu)} x_{i}^{\left(y_{i}\right.}
$$

which shows that $f^{(r)}$ is a skew-symmetric non-degenerate bilinear form on $R^{n(r+1)}$.

Proposition 11. 4. If $G=S_{p}(m, f)$, then $G^{(r)} \subset S_{p}\left(m(r+1), f^{(r)}\right)$.

Proof. Similar to the of Proposition 11. 1.

By the same arguments as the proof of Th. 6. 6 [4] we obtain the following

Theorem 11. 5. If a manifold $M$ has a (resp. an almost) symplectic struct ure then $\stackrel{r}{T} M$ has a canonical (almost) symplectic structure.

(III) $G=G L(V, W)$.

We have the following Proposition whose proof will be omitted.

Proposition 11. 6. If a manifold $M$ has a k-dimensional (completely integrable) differential system, then $\stackrel{r}{T} M$ has a canonical $k(r+1)$-dimensional (completely integrable) differential system.

(IV) $G=O(k, n-k)$.

Let $g$ be a symmetric non-degenerate bilinear form on $R^{n}$ of signature $(k, n-k)$ and let $\pi_{r}: \stackrel{r}{T} R \rightarrow R$ be the same projection as in (II) and let $g^{(r)}$ be the map $g^{(r)}=\pi_{r} \circ(\stackrel{r}{T} g): \stackrel{r}{T} R^{n} \times \stackrel{r}{T} R^{n} \rightarrow R$. We denote by $O(k, n-k, g)$ or simply $O(g)$ the group of all $a \in G L(n)$ such that $a$ leaves $g$ invariant.

LAMma 11. 7. The notations being as above, $g^{(r)}$ is a symmetric nondegenerate bilinear form on $R^{n(r+1)}$ of signature $(n(r+1) / 2, n(r+1) / 2)$ if $r$ is odd and of signature $\left(k+\frac{r n}{2}, n-k+\frac{r n}{2}\right)$ if $r$ is even.

Proof. If the bilinear form $g$ is expressed by a symmetric matrix $A=\left(a_{j}^{i}\right) \in G L(n)$, then by the same computation as the proof of (11.1) in Lemma 11. 3, we see that $g^{(r)}$ is expressed by the following matrix 


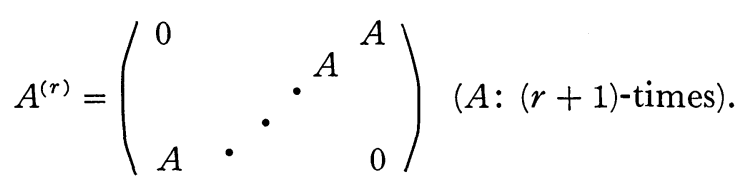

Since $A$ is of signature $(k, n-k), A^{(r)}$ is of signature $(n(r+1) / 2, n(r+1) / 2)$ if $r$ is odd and of signature $(k+(r n / 2), n-k+(r n / 2))$ if $r$ is even.

$$
\text { Q.E.D. }
$$

LemmA 11. 8. If $G=O(g)$, then $G^{(r)} \subset O\left(g^{(r)}\right)$, the signature of $g^{(r)}$ being given in Lemma 11. 7.

\section{Proof. Omitted.}

By the Lemma 11. 8, we obtain the following

Theorem 11. 9. If $M$ has a pseudo-Riemannian metric, then $\stackrel{r}{T} M$ has a canonical pseudo-Riemannian metric for every $r$.

(V) $G=G L(n, C) \times 1 \subset G L(2 n+1)$.

Lemma 11. 10. Let $G=G L(n, C) \times 1 \subset G L(2 n+1)$. Then, $G^{(r)} \subset G L((2 n+1)$ $(r+1) / 2, C)$ if $r$ is odd and $G^{(r)} \subset G L((2 n r+2 n+r) / 2, C) \times 1$ if $r$ is even.

Proof. We shall omit the proof, which is similar to the proof of Lemma 6. 14 [4].

By Lemma 11. 10. we obtain the following

THEOREM 11. 11. If $M$ has an almost contact structure, then (i) $\stackrel{r}{T} M$ has a canonical almost complex structure for any odd $r$ and (ii) $\stackrel{r}{T} M$ has a canonical almost contact structure for even $r$.

\section{REFERENCES}

[1] D. Bernard, Sur la géometrie différentielle des $G$-structures, Ann. Inst. Fourier 10 (1960), 151-270.

[2 ] S.S. Chern, The geometry of $G$-structures, Bull. Amer. Math. Soc. 72 (1966), 167-219.

[ 3 ] S. Kobayashi, Theory of connections, Ann. Mat. Pura Appl., 43 (1957), 119-194.

[4] A. Morimoto, Prolongations of $G$-structures to tangent bundles, Nagoya Math. J. 32 (1968), 67-108.

[ 5 ] N. Steenrod, The topology of fibre bundles, Princeton Univ. Press 1951.

[6] S. Sternberg, Lectures on Differential Geometry, Englewood Cliffs 1964.

[ 7 ] K. Yano-S. Ishihara, Differential geometry of tangent bundles of order 2, to appear.

\section{Nagoya University}

